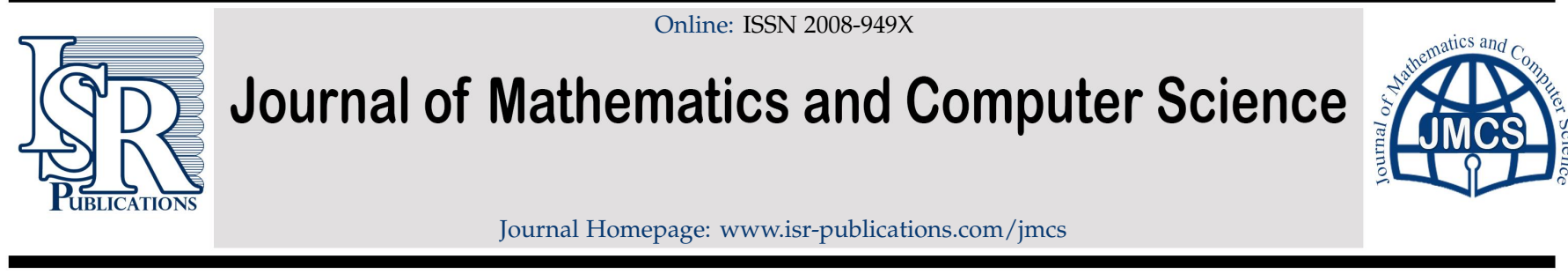

\title{
Quadrature method with exponential fitting for delay differ- ential equations having Layer behavior
}

\author{
M. Lalu, K. Phaneendra* \\ Department of Mathematics, University College of Engineering, Osmania University, Hyderabad, India.
}

\begin{abstract}
In this paper, we suggest a computational method for solving delay differential equations, with one end layer, dual layer and interior layer behavior using Gaussian quadrature. The problem is initially replaced with the analogous first order neutral type delay differential equation by taking the perturbation parameter within the differentiated term. For the numerical solution with boundary layer at one endpoint, dual boundary layers and internal boundary layers, the Gaussian two-point quadrature scheme was extracted with exponential fitting. Using model examples, the suggested approach is used for various perturbation and delay parameter values. The numerical scheme is validated and supported by the comparison of maximum errors with the other results in the literature. Convergence of the method is examined. For various delay parameter values, the layer structure is depicted in graphs.
\end{abstract}

Keywords: Delay differential equations, boundary layer, Gauss quadrature two point formula, dual layer, internal layer, fitting factor.

2020 MSC: 65L10, 65L11, 65L12.

(C)2022 All rights reserved.

\section{Introduction}

The modeling of a physical system in the theory of control, increase the order and stiffness of these systems by the existence of small time parasitic parameters such as resistance, inductance, capacitance and moments of inertia. The elimination of these constants leads to a depletion in the system order. These problems are identified as singular perturbation problems and when such systems are taken into consideration, the physical system's earlier history as well as the present state are examined. Then such problems are named as singularly perturbed differential delay equations. These problems occur in firstexit neurobiology problems and in the formulation of various biological processes in the biosciences.

Researchers can refer to Derstine et al. [2], Elsgolt's [4], Stein [14], Tuckwell [15], Longtin and Milton [9], etc for further study of mathematical aspects of the above class of models. For the various analytical and numerical methods for the solution of singular perturbation problems, one can refer to [3, 5]. In [1], researchers proposed a technique for the solution of second order boundary value problem using spline method and collocation along with the shooting method. A finite difference scheme [6] is presented for

\footnotetext{
*Corresponding author

Email addresses: lalunaikmudavathou@gmail.com (M. Lalu), kollojuphaneendra@yahoo.co.in (K. Phaneendra)
}

doi: $10.22436 /$ jmcs.025.02.08

Received: 2021-01-11 Revised: 2021-03-10 Accepted: 2021-05-01 
the numerical result of layer behavior problems with delay term. Here, the researchers initially reduced the problem of delay to a boundary value problem by allowing the delay term outside, then employed a difference scheme to solve the problem.

In [13], the authors suggested a method of computation to solve problems with layer. They have implemented rule of Simpson on the neutral type first order differential equation, which is reduced from the original problem. In [8], the authors used asymptotic matching expansion and Laplace transforms to analyze the layer behavior of linear second-order differential-difference equations with small shifts having layer behavior. In [7], the WKB approach modified by Lange and Miura is used in combination with the matched asymptotic expansions to analyze the differential-difference boundary value problems with rapid oscillation solutions.

\section{Description of the numerical scheme}

Consider the boundary value problem with delay in singularly perturbed differential equation

$$
\varepsilon w^{\prime \prime}(s)+\mathrm{P}(\mathrm{s}) w^{\prime}(\mathrm{s}-\delta)+\mathrm{Q}(\mathrm{s}) w(\mathrm{~s})=\mathrm{F}(\mathrm{s}),
$$

with boundary conditions

$$
w(s)=\phi(s), \quad-\delta \leqslant s \leqslant u \text { and } w(v)=\beta .
$$

Here $0<\varepsilon \ll 1$ is a perturbation parameter, $\delta$ also a small delay or shift. The functions $\mathrm{P}(\mathrm{s}), \mathrm{Q}(\mathrm{s})$, $\mathrm{F}(\mathrm{s})$ and $\phi(\mathrm{s})$ are sufficiently differentiable functions and $\beta$ is a constant. When delay is zero, Eq. (2.1) turns to a problem of singular perturbation, displays turning points and layers subject to the convection coefficient. The behavior of the layer is preserved only for $\delta \neq 0$ and $\delta$ is of $o(\varepsilon)$. Since the solution $w(s)$ of the problem Eq. (2.1) with Eq. (2.2) is sufficiently differentiable, the retarded term $w^{\prime}(s-\delta)$ is expanded in Taylor series, then we get

$$
w^{\prime}(s-\delta) \approx w^{\prime}(s)-\delta w^{\prime \prime}(s) .
$$

Using Eq. (2.3) in Eq. (2.1), we have

$$
\begin{gathered}
(\varepsilon-\delta \mathrm{P}(\mathrm{s})) w^{\prime \prime}(\mathrm{s})+\mathrm{P}(\mathrm{s}) w^{\prime}(\mathrm{s})+\mathrm{Q}(\mathrm{s}) w(\mathrm{~s})=\mathrm{F}(\mathrm{s}) \\
\varepsilon\left(1-\frac{\delta \mathrm{P}(\mathrm{s})}{\varepsilon}\right) w^{\prime \prime}(\mathrm{s})+\mathrm{P}(\mathrm{s}) w^{\prime}(\mathrm{s})+\mathrm{Q}(\mathrm{s}) w(\mathrm{~s})=\mathrm{F}(\mathrm{s})
\end{gathered}
$$

Eq. (2.4) is altered into singularly perturbed boundary value problem of the form

$$
\varepsilon w^{\prime \prime}(\mathrm{s})+\widetilde{\mathrm{P}}(\mathrm{s}) \boldsymbol{w}^{\prime}(\mathrm{s})+\widetilde{\mathrm{Q}}(\mathrm{s}) w(\mathrm{~s})=\widetilde{\mathrm{F}}(\mathrm{s}), \quad \mathrm{u} \leqslant \mathrm{s} \leqslant v,
$$

with $w(u)=\phi_{0}, w(v)=\beta$. Here $\widetilde{P}(s)=\frac{\mathrm{P}(\mathrm{s})}{\left(1-\frac{\mathrm{P}(\mathrm{s}) \mathcal{\delta}}{\varepsilon}\right)}, \widetilde{\mathrm{Q}}(\mathrm{s})=\frac{\mathrm{Q}(\mathrm{s})}{\left(1-\frac{\mathrm{P}(\mathrm{s}) \mathcal{\delta}}{\varepsilon}\right)}, \widetilde{\mathrm{F}}(\mathrm{s})=\frac{\mathrm{F}(\mathrm{s})}{\left(1-\frac{\mathrm{P}(\mathrm{s}) \mathcal{\delta}}{\varepsilon}\right)}$.

\subsection{Boundary layer at Left-end}

If in Eq. (2.5), $\widetilde{P}(s) \geqslant M>0$ all over the domain $[u, v]$, where $M$ is positive constant, then layer will be in the vicinity of $s=u$. Using Taylor's expansion, we have

$$
w^{\prime}(s-\varepsilon) \approx w^{\prime}(s)-\varepsilon w^{\prime \prime}(s),
$$

implies

$$
\varepsilon w^{\prime \prime}(s)=w^{\prime}(s)-w^{\prime}(s-\varepsilon)
$$


Accordingly, Eq. (2.5) is substituted by the following first order differential equation having perturbation parameter inside the differentiated term

$$
w^{\prime}(s)=w^{\prime}(s-\varepsilon)-\widetilde{P}(s) w^{\prime}(s)-\widetilde{Q}(s) w(s)+\widetilde{F}(s) .
$$

The domain $[u, v]$ is partitioned into $N$ equal subregions of mesh size $h=\frac{v-u}{N}$ so that $s_{i}=u+i h, i=$ $0,1, \ldots, N+1$ are the grid points. Eq. (2.6) is integrated with respect to from $s_{i}$ to $s_{i+1}$, then we have

$$
\begin{aligned}
\int_{s_{i}}^{s_{i+1}} w^{\prime}(s) d s & =\int_{s_{i}}^{s_{i+1}} w^{\prime}(s-\varepsilon)-\widetilde{P}(s) w^{\prime}(s)-\widetilde{Q}(s) w(s)+\widetilde{F}(s) d s \\
w\left(s_{i+1}\right)-w\left(s_{i}\right) & =\int_{s_{i}}^{s_{i+1}} w^{\prime}(s-\varepsilon) d s-\int_{s_{i}}^{s_{i+1}} \widetilde{P}(s) w^{\prime}(s) d s-\int_{s_{i}}^{s_{i+1}} \widetilde{Q}(s) w(s) d s+\int_{s_{i}}^{s_{i+1}} \widetilde{F}(s) d s .
\end{aligned}
$$

Using two-point Gaussian quadrature formula, we have

$$
\int_{-1}^{1} \mathcal{G}(\mathrm{s}) \mathrm{ds}=\mathcal{G}\left(\frac{1}{\sqrt{3}}\right)+\mathcal{G}\left(\frac{-1}{\sqrt{3}}\right)
$$

For any function $\mathcal{G}$, differentiable in an arbitrary interval $\left[s_{i}, s_{i+1}\right]$, the two-point Gaussian quadrature formula gives

$$
\int_{s_{i}}^{s_{i+1}} \mathcal{G}(s) d s=\frac{h}{2}\left(\mathcal{G}\left(s_{i}+\bar{k}\right)+\mathcal{G}\left(s_{i+1}-\bar{k}\right)\right)
$$

where $\bar{k}=\frac{h\left(1-\frac{1}{\sqrt{3}}\right)}{2}$. Using Eq. (2.8) in Eq. (2.7), we get

$$
\begin{aligned}
w_{i+1}-w_{i}= & w\left(s_{i+1}-\varepsilon\right)-w\left(s_{i}-\varepsilon\right)-\widetilde{P}\left(s_{i+1}\right) w\left(s_{i+1}\right)+\widetilde{P}\left(s_{i}\right) w\left(s_{i}\right) \\
& +\frac{h}{2}\left[\widetilde{\mathrm{P}}^{\prime}\left(s_{i+1}-\bar{k}\right) w\left(s_{i+1}-\bar{k}\right)+\widetilde{P}^{\prime}\left(s_{i}+\bar{k}\right) w\left(s_{i}+\bar{k}\right)\right] \\
& -\frac{h}{2}\left[\widetilde{Q}\left(s_{i+1}-\bar{k}\right) w\left(s_{i+1}-\bar{k}\right)+\widetilde{Q}\left(s_{i}+\bar{k}\right) w\left(s_{i}+\bar{k}\right)\right] \\
& +\frac{h}{2}\left[\widetilde{\mathrm{F}}\left(s_{i+1}-\bar{k}\right)+\widetilde{F}\left(s_{i}+\bar{k}\right)\right] .
\end{aligned}
$$

Using the linear interpolation for $w\left(s_{i+1}-\varepsilon\right), w\left(s_{i}-\varepsilon\right), w\left(s_{i}-\bar{k}\right)$ and $w\left(s_{i+1}-\bar{k}\right)$ Eq. (2.9) reduces to

$$
\begin{aligned}
& \left\{\frac{\varepsilon}{h}+\widetilde{\mathrm{P}}^{\prime}\left(s_{i}+\bar{k}\right) \frac{\bar{k}}{2}-\widetilde{\mathrm{Q}}\left(s_{i}+\overline{\mathrm{k}}\right) \frac{\overline{\mathrm{k}}}{2}\right\} w_{i-1} \\
& \quad+\left\{\frac{-2 \varepsilon}{\mathrm{h}}-\widetilde{\mathrm{P}}\left(s_{i}\right)-\widetilde{\mathrm{P}}^{\prime}\left(s_{i+1}-\overline{\mathrm{k}}\right) \frac{\overline{\mathrm{k}}}{2}-\widetilde{\mathrm{P}}^{\prime}\left(s_{i}+\overline{\mathrm{k}}\right)\left(\frac{\mathrm{h}+\overline{\mathrm{k}}}{2}\right)+\widetilde{\mathrm{Q}}\left(s_{i+1}-\overline{\mathrm{k}}\right) \frac{\overline{\mathrm{k}}}{2}+\widetilde{\mathrm{Q}}\left(s_{i}+\overline{\mathrm{k}}\right)\left(\frac{\mathrm{h}+\overline{\mathrm{k}}}{2}\right)\right\} w_{i} \\
& \quad+\left\{\frac{\varepsilon}{\mathrm{h}}-\widetilde{\mathrm{P}}^{\prime}\left(s_{i+1}-\overline{\mathrm{k}}\right)\left(\frac{\mathrm{h}-\overline{\mathrm{k}}}{2}\right)+\widetilde{\mathrm{P}}\left(s_{i+1}\right)+\widetilde{\mathrm{Q}}\left(s_{i}+\overline{\mathrm{k}}\right)\left(\frac{\mathrm{h}-\overline{\mathrm{k}}}{2}\right)\right\} w_{i+1} \\
& =\frac{h}{2}\left\{\widetilde{\mathrm{F}}\left(s_{i+1}-\overline{\mathrm{k}}\right)+\widetilde{\mathrm{F}}\left(s_{i}+\overline{\mathrm{k}}\right)\right\} .
\end{aligned}
$$

Rearranging this equation, we have

$$
\begin{aligned}
& \frac{\varepsilon}{h^{2}}\left(w_{i-1}-2 w_{i}+w_{i+1}\right)+\left\{\frac{\widetilde{P}^{\prime}\left(s_{i}+\bar{k}\right)\left(\frac{\bar{k}}{2}\right)-\widetilde{Q}\left(s_{i}+\bar{k}\right)\left(\frac{\bar{k}}{2}\right)}{h}\right\} w_{i-1} \\
& \quad+\left\{\frac{-\widetilde{P}\left(s_{i}\right)-\widetilde{p}^{\prime}\left(s_{i+1}-\bar{k}\right) \frac{\bar{k}}{2}-\widetilde{P}^{\prime}\left(s_{i}+\bar{k}\right)\left(\frac{h+\bar{k}}{2}\right)+\widetilde{Q}\left(s_{i+1}-\bar{k}\right) \frac{\widetilde{k}}{2}+\widetilde{Q}\left(s_{i}+\bar{k}\right)\left(\frac{h+\bar{k}}{2}\right)}{h}\right\} w_{i}
\end{aligned}
$$




$$
+\left\{\frac{-\widetilde{P}^{\prime}\left(s_{i+1}-\bar{k}\right)\left(\frac{h-\bar{k}}{2}\right)+\widetilde{P}\left(s_{i+1}\right)+\widetilde{Q}\left(s_{i+1}-\bar{k}\right)\left(\frac{h-\bar{k}}{2}\right)}{h}\right\} w_{i+1}=\left\{\frac{\widetilde{F}\left(s_{i+1}+\bar{k}\right)+\widetilde{F}\left(s_{i}+\bar{k}\right)}{2}\right\} .
$$

Introducing a fitting factor $\widetilde{\sigma}(\widetilde{\rho})$ in this scheme, to control the layer behaviour due the small values of perturbation parameter $\varepsilon$, we get

$$
\begin{aligned}
& \frac{\widetilde{\sigma} \varepsilon}{h^{2}}\left(w_{i-1}-2 w_{i}+w_{i+1}\right)+\left\{\frac{\widetilde{P}^{\prime}\left(s_{i}+\bar{k}\right)\left(\frac{\bar{k}}{2}\right)-\widetilde{Q}\left(s_{i}+\bar{k}\right)\left(\frac{\bar{k}}{2}\right)}{h}\right\} w_{i-1} \\
& \quad+\left\{\frac{-\widetilde{P}\left(s_{i}\right)-\widetilde{P}^{\prime}\left(s_{i+1}-\bar{k}\right) \frac{\bar{k}}{2}-\widetilde{P}^{\prime}\left(s_{i}+\bar{k}\right)\left(\frac{h+\bar{k}}{2}\right)+\widetilde{Q}\left(s_{i+1}-\bar{k}\right) \frac{\bar{k}}{2}+\widetilde{Q}\left(s_{i}+\bar{k}\right)\left(\frac{h+\bar{k}}{2}\right)}{h}\right\} w_{i} \\
& \quad+\left\{\frac{-\widetilde{P}^{\prime}\left(s_{i+1}-\bar{k}\right)\left(\frac{h-\bar{k}}{2}\right)+\widetilde{P}\left(s_{i+1}\right)+\widetilde{Q}\left(s_{i+1}-\bar{k}\right)\left(\frac{h-\bar{k}}{2}\right)}{h}\right\} w_{i+1} \\
& =\left\{\frac{\widetilde{F}\left(s_{i+1}+\bar{k}\right)+\widetilde{F}\left(s_{i}+\bar{k}\right)}{2}\right\} .
\end{aligned}
$$

The value of $\widetilde{\sigma}(\widetilde{\rho})$ is obtained by the procedure given by Doolan et al. [3] and is given by

$$
\widetilde{\sigma}(\widetilde{\rho})=\frac{s_{0} e^{-\mathcal{D}_{0}}}{\sinh \left(\mathcal{D}_{0}\right)}, \quad \text { where } \quad \mathcal{D}_{0}=\left(\frac{\widetilde{P}(0) \widetilde{\rho}}{2}\right), \widetilde{\rho}=\frac{h}{\varepsilon} .
$$

Eq. (2.10) can be revised in a recurrence relation as

$$
\widetilde{\mathrm{A}}_{i} w_{i-1}-\widetilde{\mathrm{B}}_{i} w_{i}+\widetilde{\mathrm{C}}_{i} w_{i+1}=\widetilde{\mathrm{R}}_{i}, i=1,2, \ldots, \mathrm{N}-1,
$$

where

$$
\begin{aligned}
& \widetilde{\mathrm{A}}_{i}=\frac{\widetilde{\sigma}(\widetilde{\rho}) \varepsilon}{h}+\widetilde{\mathrm{P}}^{\prime}\left(s_{i}+\overline{\mathrm{k}}\right) \frac{\bar{k}}{2}-\widetilde{\mathrm{Q}}\left(s_{i+1}+\overline{\mathrm{k}}\right) \frac{\bar{k}}{2} \\
& \widetilde{\mathrm{B}}_{i}=\frac{2 \widetilde{\sigma}(\widetilde{\rho}) \varepsilon}{\mathrm{h}}+\widetilde{\mathrm{P}}\left(s_{i}\right)+\widetilde{\mathrm{P}}^{\prime}\left(s_{i+1}-\overline{\mathrm{k}}\right) \frac{\bar{k}}{2}+\widetilde{\mathrm{P}}^{\prime}\left(s_{i}-\overline{\mathrm{k}}\right) \mathrm{h}_{1}-\widetilde{\mathrm{Q}}\left(s_{i+1}+\bar{k}\right)\left(\frac{\bar{k}}{2}\right)-\widetilde{\mathrm{Q}}\left(s_{i}+\bar{k}\right) h_{1}, \\
& \widetilde{\mathrm{C}}_{i}=\frac{\widetilde{\sigma}(\widetilde{\rho}) \varepsilon}{h}-\widetilde{\mathrm{P}}^{\prime}\left(s_{i+1}-\overline{\mathrm{k}}\right) \mathrm{h}_{2}+\widetilde{\mathrm{P}}\left(s_{i+1}\right)+\widetilde{\mathrm{Q}}\left(s_{i+1}-\bar{k}\right) h_{2}, \\
& \widetilde{\mathrm{R}}_{i}=\frac{h}{2}\left[\widetilde{\mathrm{F}}\left(s_{i+1}-\overline{\mathrm{k}}\right)+\widetilde{\mathrm{F}}\left(s_{i}+\overline{\mathrm{k}}\right)\right], \text { here } h_{1}=\left(\frac{h+\bar{k}}{2}\right), h_{2}=\left(\frac{h-\bar{k}}{2}\right) .
\end{aligned}
$$

The system Eq. (2.11) is solved efficiently using the Thomas Algorithm.

\subsection{Boundary layer at right-end}

If in Eq. (2.5), $\widetilde{P}(s) \leqslant \widetilde{M}<0$ over the domain $[u, v]$, where $\widetilde{M}$ is negative value, then layer exists in the vicinity of $s=v$. Taylor series expansion of $w^{\prime}(s+\varepsilon)$ gives

$$
w^{\prime}(s+\varepsilon) \approx w^{\prime}(s)+\varepsilon w^{\prime \prime}(s),
$$

implies

$$
\varepsilon w^{\prime \prime}(s) \approx w^{\prime}(s+\varepsilon)-w^{\prime}(s),
$$

and subsequently, Eq. (2.5) is substituted by the following first order differential equation

$$
w^{\prime}(\mathrm{s})=w^{\prime}(\mathrm{s}+\varepsilon)+\widetilde{\mathrm{P}}(\mathrm{s}) w^{\prime}(\mathrm{s})+\widetilde{\mathrm{Q}}(\mathrm{s}) w(\mathrm{~s})-\widetilde{\mathrm{F}}(\mathrm{s}) .
$$


Integrating Eq. (2.12) on $\left[s_{i-1}, s_{i}\right]$ we get

$$
w\left(s_{i}\right)-w\left(s_{i-1}\right)=\int_{s_{i-1}}^{s_{i}} w^{\prime}(s+\varepsilon) d s+\int_{s_{i-1}}^{s_{i}} \widetilde{P}(s) w^{\prime}(s) d s+\int_{s_{i-1}}^{s_{i}} \widetilde{Q}(s) w(s) d s-\int_{s_{i-1}}^{s_{i}} \widetilde{\mathrm{F}}(s) d s .
$$

The two-point Gaussian quadrature formula for any differentiable function $\mathcal{G}$ in an arbitrary interval $\left[s_{i-1}, s_{i}\right]$ gives

$$
\int_{s_{i-1}}^{s_{i}} \mathcal{G}(s) d s=\frac{h}{2}\left(\mathcal{G}\left(s_{i-1}+\bar{k}\right)+\mathcal{G}\left(s_{i}-\bar{k}\right)\right) .
$$

Using Eq. (2.14) in the Eq. (2.13) becomes

$$
\begin{aligned}
w\left(s_{i}\right)-w\left(s_{i-1}\right)= & w\left(s_{i-1}-\varepsilon\right)-w\left(s_{i}+\varepsilon\right)+\widetilde{P}\left(s_{i-1}\right) w\left(s_{i-1}\right)-\widetilde{\mathrm{P}}\left(s_{i}\right) w\left(s_{i}\right) \\
& -\frac{h}{2}\left[\widetilde{\mathrm{P}}^{\prime}\left(s_{i-1}-\overline{\mathrm{k}}\right) w\left(s_{i-1}-\overline{\mathrm{k}}\right)+\widetilde{\mathrm{P}}^{\prime}\left(s_{i}+\overline{\mathrm{k}}\right) w\left(s_{i}+\overline{\mathrm{k}}\right)\right] \\
& +\frac{\mathrm{h}}{2}\left[\widetilde{\mathrm{Q}}\left(s_{i-1}-\overline{\mathrm{k}}\right) w\left(s_{i-1}-\overline{\mathrm{k}}\right)+\widetilde{\mathrm{Q}}\left(s_{i}+\overline{\mathrm{k}}\right) w\left(s_{i}+\overline{\mathrm{k}}\right)\right] \\
& -\frac{\mathrm{h}}{2}\left[\widetilde{\mathrm{F}}\left(s_{i-1}-\overline{\mathrm{k}}\right)+\widetilde{\mathrm{F}}\left(s_{i}+\overline{\mathrm{k}}\right)\right] .
\end{aligned}
$$

Using linear interpolation for the terms in the above equation, $w\left(s_{i-1}-\varepsilon\right), w\left(s_{i}+\varepsilon\right), w\left(s_{i-1}-\bar{k}\right)$, and $w\left(s_{i}-\bar{k}\right)$ we get

$$
\begin{aligned}
& \left\{\frac{\varepsilon}{h}-\widetilde{P}\left(s_{i-1}\right)-\widetilde{P}^{\prime}\left(s_{i-1}+\bar{k}\right) h_{1}+\widetilde{Q}\left(s_{i-1}+\bar{k}\right) h_{1}\right\} w_{i-1} \\
& +\left\{\frac{-2 \varepsilon}{h}+\widetilde{P}\left(s_{i}\right)-\widetilde{P}^{\prime}\left(s_{i}-\bar{k}\right) h_{2}-\widetilde{P}^{\prime}\left(s_{i-1}+\bar{k}\right)\left(\frac{\widetilde{k}}{2}\right)+\widetilde{Q}\left(s_{i}-\bar{k}\right) h_{2}+\widetilde{Q}\left(s_{i-1}+\bar{k}\right)\left(\frac{\bar{k}}{2}\right)\right\} w_{i} \\
& +\left\{\frac{\varepsilon}{h}+\widetilde{P}^{\prime}\left(s_{i}-\bar{k}\right)\left(\frac{\bar{k}}{2}\right)-\widetilde{Q}\left(s_{i}-\bar{k}\right)\left(\frac{\bar{k}}{2}\right)\right\} w_{i+1} \\
& =\frac{h}{2}\left\{\widetilde{F}\left(s_{i}-\bar{k}\right)+\widetilde{F}\left(s_{i-1}+\bar{k}\right)\right\} .
\end{aligned}
$$

Here $h_{1}=\left(\frac{h-\bar{k}}{2}\right), h_{2}=\left(\frac{h+\bar{k}}{2}\right)$. Rearranging this equation, we have

$$
\begin{aligned}
& \frac{\varepsilon}{h^{2}}\left(w_{i-1}-2 w_{i}+w_{i+1}\right)+\left\{\frac{-\widetilde{P}\left(s_{i-1}\right)-\widetilde{P}^{\prime}\left(s_{i-1}+\bar{k}\right)\left(\frac{h-\bar{k}}{2}\right)+\widetilde{Q}\left(s_{i-1}+\bar{k}\right)\left(\frac{h-\bar{k}}{2}\right)}{h}\right\} w_{i-1} \\
& +\left\{\frac{\widetilde{P}\left(s_{i}\right)-\widetilde{P}^{\prime}\left(s_{i}-\bar{k}\right)\left(\frac{h+\bar{k}}{2}\right)-\widetilde{P}^{\prime}\left(s_{i-1}+\bar{k}\right)\left(\frac{\bar{k}}{2}\right)+\widetilde{Q}\left(s_{i}-\bar{k}\right)\left(\frac{h+\bar{k}}{2}\right)+\widetilde{Q}\left(s_{i-1}+\bar{k}\right)\left(\frac{\bar{k}}{2}\right)}{h}\right\} w_{i} \\
& \quad+\left\{\frac{\widetilde{P}^{\prime}\left(s_{i}-\bar{k}\right)\left(\frac{\bar{k}}{2}\right)-\widetilde{Q}\left(s_{i}-\bar{k}\right)\left(\frac{\bar{k}}{2}\right)}{h}\right\} w_{i+1}=\left\{\frac{\widetilde{F}\left(s_{i}-\bar{k}\right)+\widetilde{F}\left(s_{i-1}+\bar{k}\right)}{2}\right\} .
\end{aligned}
$$

To handle the layer behaviour at right end $s=v$, for the small values of the perturbation parameter, inserting a fitting factor $\widetilde{\sigma}(\widetilde{\rho})$ in the above scheme, we get

$$
\frac{\widetilde{\sigma} \varepsilon}{h^{2}}\left(w_{i-1}-2 w_{i}+w_{i+1}\right)+\left\{\frac{-\widetilde{P}\left(s_{i-1}\right)-\widetilde{P}^{\prime}\left(s_{i-1}+\bar{k}\right)\left(\frac{h-\bar{k}}{2}\right)+\widetilde{Q}\left(s_{i-1}+\bar{k}\right)\left(\frac{h-\bar{k}}{2}\right)}{h}\right\} w_{i-1}
$$




$$
\begin{aligned}
& +\left\{\frac{\widetilde{P}\left(s_{i}\right)-\widetilde{P}^{\prime}\left(s_{i}-\bar{k}\right)\left(\frac{h+\bar{k}}{2}\right)-\widetilde{P}^{\prime}\left(s_{i-1}+\bar{k}\right)\left(\frac{\bar{k}}{2}\right)+\widetilde{Q}\left(s_{i}-\bar{k}\right)\left(\frac{h+\bar{k}}{2}\right)+\widetilde{Q}\left(s_{i-1}+\bar{k}\right)\left(\frac{\bar{k}}{2}\right)}{h}\right\} w_{i} \\
& +\left\{\frac{\widetilde{P}^{\prime}\left(s_{i}-\widetilde{k}\right)\left(\frac{\bar{k}}{2}\right)-\widetilde{Q}\left(s_{i}-\bar{k}\right)\left(\frac{\bar{k}}{2}\right)}{h}\right\} w_{i+1}=\left\{\frac{\widetilde{F}\left(s_{i}-\bar{k}\right)+\widetilde{F}\left(s_{i-1}+\bar{k}\right)}{2}\right\} .
\end{aligned}
$$

The value of $\widetilde{\sigma}(\widetilde{\rho})$ is obtained by the procedure given by Doolan et al. [3] and is given by

$$
\widetilde{\sigma}(\widetilde{\rho})=\frac{\widetilde{\rho} \widetilde{P}(0) e^{\mathcal{D}_{N}}}{2 \sinh \left(\mathcal{D}_{N}\right)} \text {, where } \mathcal{D}_{N}=\left(\frac{\widetilde{P}(N) \widetilde{\rho}}{2}\right), \widetilde{\rho}=\frac{h}{\varepsilon} \text {. }
$$

Eq. (2.16) simplified into the tridiagonal system

$$
\tilde{\mathrm{A}}_{i} w_{i-1}-\tilde{\mathrm{B}}_{i} w_{i}+\tilde{\mathrm{C}}_{i} w_{i+1}=\tilde{\mathrm{R}}_{i} \text { for } i=1,2, \ldots, \mathrm{N}-1,
$$

where

$$
\begin{aligned}
& \widetilde{A}_{i}=\left\{\frac{\widetilde{\sigma}(\widetilde{\rho}) \varepsilon}{h}-\widetilde{P}\left(s_{i-1}\right)-\widetilde{P}^{\prime}\left(s_{i-1}+\bar{k}\right)\left(\frac{h-\bar{k}}{2}\right)+\widetilde{Q}\left(s_{i-1}+\bar{k}\right)\left(\frac{h-\bar{k}}{2}\right)\right\}, \\
& \widetilde{B}_{i}=\left\{\frac{2 \widetilde{\sigma}(\widetilde{\rho}) \varepsilon}{h}-\widetilde{P}\left(s_{i}\right)+\widetilde{P}^{\prime}\left(s_{i}+\bar{k}\right) h_{2}+\widetilde{P}^{\prime}\left(s_{i-1}+\bar{k}\right)\left(\frac{\bar{k}}{2}\right)-\widetilde{Q}\left(s_{i}-\bar{k}\right) h_{2}-\widetilde{Q}\left(s_{i-1}+\bar{k}\right)\left(\frac{\bar{k}}{2}\right)\right\}, \\
& \widetilde{C}_{i}=\left\{\frac{\widetilde{\sigma}(\widetilde{\rho}) \varepsilon}{h}+\widetilde{P}^{\prime}\left(s_{i}-\bar{k}\right)\left(\frac{\bar{k}}{2}\right)-\widetilde{Q}\left(s_{i}-\bar{k}\right)\left(\frac{\bar{k}}{2}\right)\right\}, \\
& \widetilde{R}_{i}=\frac{h}{2}\left[\widetilde{F}\left(s_{i}-\bar{k}\right)+\widetilde{F}\left(s_{i-1}+\bar{k}\right)\right] .
\end{aligned}
$$

The system Eq. (2.17) is solved with Thomas algorithm.

\subsection{Dual boundary layer problem}

With the following assumptions

$$
\begin{aligned}
& \widetilde{\mathrm{P}}\left(\mathrm{s}_{\mathrm{m}}\right)=0, \widetilde{\mathrm{P}}^{\prime}\left(\mathrm{s}_{\mathrm{m}}\right)<0, \text { where } \mathrm{s}_{\mathrm{m}}=\frac{\mathrm{u}+v}{2}, \\
& |\widetilde{\mathrm{P}}(\mathrm{s})| \geqslant \widetilde{\mathrm{P}}_{0}>0 \text { for } \mathrm{u} \leqslant \mathrm{s} \leqslant v, \\
& \widetilde{\mathrm{Q}}(\mathrm{s}) \leqslant \widetilde{\mathrm{Q}}_{0}<0, \quad \forall \mathrm{s} \in[\mathrm{u}, v], \\
& \left|\widetilde{\mathrm{P}}^{\prime}(\mathrm{s})\right| \geqslant \frac{\left|\widetilde{\mathrm{P}}^{\prime}(\mathrm{m})\right|}{2}, \quad \forall \mathrm{s} \in[\mathrm{u}, v],
\end{aligned}
$$

the problem (2.1)-(2.2) has unique solution, expressing exponential boundary layers at both end points $s=u$ and $s=v$. Discretize the interval $[u, v]$ into $N$ equal parts with mesh size $h=\frac{1}{N}$ so that $\theta_{i}=u+i h$ for $i=1,2, \ldots, N$ are the mesh points. Denote $\frac{N}{2}=m$. Then, divide the interval $[u, v]$ into sub intervals $\left[s_{i-1}, s_{i}\right]$ for $i=1,2, \ldots, m$ and $\left[s_{i}, s_{i+1}\right]$ for $i=m+1, m+2, \ldots, N-1$. We derive the numerical method in the two subintervals $\left[u, s_{m}\right]$ and $\left[s_{m}, v\right]$ where in $\left[u, s_{m}\right]$ layer will be at left end point $\theta=u$ whereas in $\left[s_{m}, v\right]$ layer exits at $s=v$. Hence, in $\left[u, s_{m}\right]$, we use the finite difference scheme Eq. (2.11) for $i=1,2, \ldots, m-1$ and in $\left[s_{m}, v\right]$ for $i=m+1, m+2, \ldots, N-1$. The scheme Eq. (2.17) is employed to get the solution. Now at $s=s_{m}$, i.e., for $i=m$, Eq. (2.1) becomes

$$
\varepsilon w^{\prime \prime}\left(s_{\mathrm{m}}\right)+\widetilde{\mathrm{Q}}\left(\mathrm{s}_{\mathrm{m}}\right) w\left(s_{\mathrm{m}}\right)=\widetilde{\mathrm{F}}\left(\mathrm{s}_{\mathrm{m}}\right) .
$$

Using the central finite difference on Eq. (2.18) at this point, the difference equation of Eq. (2.18) is

$$
\widetilde{A}_{m} w_{m-1}+\widetilde{B}_{m i} w_{m}+\widetilde{C}_{m} w_{m+1}=\widetilde{F}_{m} \text {. }
$$

Here $\widetilde{A}_{m}=\frac{\widetilde{\sigma} \varepsilon}{h^{2}}, \widetilde{B}_{m}=\frac{2 \widetilde{\sigma} \varepsilon}{h^{2}}-\widetilde{Q}_{m}$, and $\widetilde{C}_{m}=\frac{\widetilde{\sigma} \varepsilon}{h^{2}}$. Now, we solve the system of Eq. (2.11), Eq. (2.19), and Eq. (2.17) using Thomas algorithm. 


\subsection{Internal boundary layer}

Under following assumptions

$$
\begin{aligned}
\widetilde{\mathrm{P}}\left(s_{\mathrm{m}}\right) & =0, \widetilde{\mathrm{P}}^{\prime}\left(\mathrm{s}_{\mathrm{m}}\right)>0, \text { where } \mathrm{s}_{\mathrm{m}}=\frac{\mathrm{u}+v}{2}, \\
\widetilde{\mathrm{Q}}(\mathrm{s}) & \leqslant \widetilde{\mathrm{Q}}_{0}<0, \\
\left|\widetilde{\mathrm{P}}^{\prime}(\mathrm{s})\right| & \geqslant \frac{\left|\widetilde{\mathrm{P}}^{\prime}(\mathrm{m})\right|}{2}, \text { for all } \mathrm{s} \in[\mathrm{u}, v],
\end{aligned}
$$

the given problem has a unique solution with interior layers at $s=s_{m}$. Decompose the interval $[u, v]$ into $N$ uniform spaced intervals with mesh size $h=\frac{1}{N}$ and with mesh points $s_{i}=u+i$ for $i=1,2, \ldots, N$. Let us denote $\frac{N}{2}=m$. Then, divide the interval $[u, v]$ into subintervals $\left[s_{i-1}, s_{i}\right]$ for $i=1,2, \ldots, m$ and $\left[s_{i}, s_{i+1}\right]$ for $i=m+1, m+2, \ldots, N-1$. For this problem, the layer is at right end point of the interval $\left[u, s_{m}\right]$ and layer is at left end point of $\left[s_{\mathfrak{m}}, v\right]$. Hence, in $\left[u, s_{\mathfrak{m}}\right]$ we use the finite difference scheme Eq. (2.17) for $i=1,2, \ldots, m-1$ and in $\left[s_{m}, v\right]$ the scheme Eq. (2.11) for $i=m+1, m+2, \ldots, N-1$ is used to get the solution. Now at $s=s_{m}$, i.e., for $i=m$, Eq. (2.1) becomes

$$
\varepsilon w^{\prime \prime}\left(s_{m}\right)+\widetilde{Q}\left(s_{m}\right) w\left(s_{m}\right)=\widetilde{F}\left(s_{m}\right) .
$$

Since there is an internal layer at $s=s_{m}$, we take average of the difference schemes Eq. (2.11) and Eq. (2.17). Hence the difference equation of Eq. (2.20) is

$$
\widetilde{\mathrm{A}}_{m} w_{m-1}+\widetilde{\mathrm{B}}_{m} w_{m}+\widetilde{\mathrm{C}}_{m} w_{m+1}=\widetilde{\mathrm{F}}_{m} .
$$

Here $\widetilde{A}_{m}=\frac{\widetilde{A}_{i L}+\widetilde{A}_{i R}}{2}, \widetilde{B}_{m}=\frac{\widetilde{B}_{i L}+\widetilde{B}_{i R}}{2}, \widetilde{C}_{m}=\frac{\widetilde{C}_{i L}+\widetilde{C}_{i R}}{2}$, and $\widetilde{F}_{m}=\frac{\widetilde{F}_{i L}+\widetilde{F}_{i R}}{2} ; \widetilde{A}_{i L}, \widetilde{B}_{i L}, \widetilde{C}_{i L}$, and $\widetilde{F}_{i L}$ denote the value of $\widetilde{A}_{i}, \widetilde{B}_{i}, \widetilde{C}_{i}$, and $\widetilde{F}_{i}$ in the left-end boundary layer case and $\widetilde{A}_{i R}, \widetilde{B}_{i R}, \widetilde{C}_{i R}$, and $\widetilde{F}_{i R}$ represents the expresiion of $\widetilde{A}_{i}, \widetilde{B}_{i}, \widetilde{C}_{i}$, and $\widetilde{F}_{i}$ in the right-end boundary layer case, respectively. Similarly, now, we solve the system of Eqs. (2.11), (2.17), and (2.21) using Thomas algorithm.

\section{Discrete invariant imbedding algorithm}

Let us set up a difference relation of the form

$$
w_{i}=Z_{i} w_{i+1}+T_{i}
$$

where $Z_{i}=Z\left(s_{i}\right)$ and $T_{i}=T\left(s_{i}\right)$ are to be determined From Eq. (3.1), we have

$$
w_{i-1}=Z_{i-1} w_{i}+T_{i-1} .
$$

Using Eq. (3.1) in Eq. (3.2) and rearranging the terms, we obtain

$$
w_{i}=\frac{\widetilde{C}_{i}}{\widetilde{B}_{i}-\widetilde{A}_{i} Z_{i-1}} w_{i+1}+\frac{\widetilde{A}_{i} T_{i-1}-\widetilde{F}_{i}}{\left(\widetilde{B}_{i}-\widetilde{A}_{i} Z_{i-1}\right)} .
$$

By comaring Eqs. (3.1) and (3.3), we get recurrence relation for $Z_{i}$ and $T_{i}$

$$
z_{i}=\frac{\widetilde{C}_{i}}{\widetilde{B}_{i}-\widetilde{A}_{i} Z_{i-1}}, \quad T_{i}=\frac{\widetilde{A}_{i} T_{i-1}-\widetilde{F}_{i}}{\left(\widetilde{B}_{i}-\widetilde{A}_{i} Z_{i-1}\right)}
$$

To solve these recurrence relation for $i=1,2,3, \ldots, N-1$, we need the initial conditions for $Z_{0}$ and $T_{0}$. By given boundary conditions, we have

$$
w_{0}=\varphi_{0}=Z_{0} w_{1}+T_{0}
$$


If we choose $w_{0}=0$, then $T_{0}=\varphi_{0}$. Now by using these initial conditions, we can compute $w_{i}$ and $T_{i}$ for $i=1,2,3, \ldots, N-1$ and using these values of $w_{i}$ and $T_{i}$ in Eq. (3.1), we obtain $w_{i}$ for $i=1,2,3, \ldots, N-1$. Under the conditions

$$
\widetilde{A}_{i}>0, \widetilde{C}_{i}>0, \quad \widetilde{B}_{i} \geqslant \widetilde{A}_{i}+\widetilde{C}_{i}, \quad\left|\widetilde{A}_{i}\right| \leqslant\left|\widetilde{C}_{i}\right|,
$$

the discrete invariant imbedding algorithm is stable.

\section{Stability analysis}

Theorem 4.1. Under the assumption that $\widetilde{\mathrm{P}}(\mathrm{s}) \geqslant \mathrm{M}>0$ and $\widetilde{\mathrm{Q}}(\mathrm{s}) \leqslant-\theta<0, \forall \mathrm{s} \in[0,1]$, the solution to the system of difference equations (2.11) together with the given boundary condition exists, is unique and satisfies

$$
\|w\|_{h, \infty} \leqslant C^{-1}\|\widetilde{R}\|_{h, \infty}+\|\phi\|_{h, \infty}+|\beta|,
$$

where $\mathrm{C}=\mathrm{M}$ or $\|\mathrm{P}\|_{\mathrm{h}, \infty}$. Here $\|.\|_{\mathrm{h}, \infty}$ is the discret $\mathrm{l}_{\infty}$-norm, given by $\|\mathrm{s}\|_{\mathrm{h}, \infty}=\max _{0 \leqslant i \leqslant N}\left|s_{i}\right|$.

Proof. Let $w_{i}$ be any mesh function satisfying $L_{1}^{N} w_{i}=\widetilde{R}_{i}$. Combining this with Eq. (2.11) followed by a rearrangement of terms gives

$$
\widetilde{\mathrm{B}}_{i} w_{i}=-\widetilde{\mathrm{R}}_{i}+\widetilde{\mathrm{A}}_{i} w_{i-1}+\widetilde{\mathrm{C}}_{i} w_{i+1} \text {. }
$$

Taking modulus on both the sides and using the non negativity of the coefficients $\left(\widetilde{A}_{i}, \widetilde{B}_{i}, \widetilde{C}_{i}\right)$, we obtain

$$
\widetilde{\mathrm{B}}_{i}\left|w_{i}\right| \leqslant\left|\widetilde{R}_{i}\right|+\widetilde{\mathrm{A}}_{i}\left|w_{i-1}\right|+\widetilde{\mathrm{C}}_{i}\left|w_{i+1}\right| \text {. }
$$

Putting the values of $\left(\widetilde{A}_{i}, \widetilde{B}_{i}, \widetilde{C}_{i}\right)$ from Eq. (2.11) in the above inequality, we get

$$
\begin{aligned}
& \left(\frac{-2 \widetilde{\sigma}(\widetilde{\rho}) \varepsilon}{h}-\widetilde{P}\left(s_{i}\right)-\widetilde{P}^{\prime}\left(s_{i+1}+\bar{k}\right) \frac{\bar{k}}{2}-\widetilde{P}^{\prime}\left(s_{i}+\bar{k}\right) h_{1}+\widetilde{Q}\left(s_{i+1}+\bar{k}\right)\left(\frac{\bar{k}}{2}\right)+\widetilde{Q}\left(s_{i}+\bar{k}\right) h_{1}\right)\left|z_{i}\right| \\
& \leqslant\left|\frac{h}{2}\left[\widetilde{F}\left(s_{i+1}-\bar{k}\right)+\widetilde{F}\left(s_{i}+\bar{k}\right)\right]\right|+\left(\frac{\widetilde{\sigma}(\widetilde{\rho}) \varepsilon}{h}+\widetilde{P}^{\prime}\left(s_{i}+\bar{k}\right) \frac{\bar{k}}{2}-\widetilde{Q}\left(s_{i+1}+\bar{k}\right) \frac{\bar{k}}{2}\right)\left|z_{i-1}\right| \\
& +\left(\frac{\widetilde{\sigma}(\widetilde{\rho}) \varepsilon}{h}-\widetilde{P}^{\prime}\left(s_{i+1}-\bar{k}\right)\left(\frac{h-\bar{k}}{2}\right)+\widetilde{P}\left(s_{i+1}\right)+\widetilde{Q}\left(s_{i+1}-k\right)\left(\frac{h-\bar{k}}{2}\right)\right)\left|z_{i+1}\right|,
\end{aligned}
$$

$\forall i=1,2,3, \ldots N-1$. A rearrangement of terms in the above in equality (4.1) yields

$$
\begin{aligned}
& \frac{\widetilde{\sigma} \varepsilon}{h^{2}}\left(\left|w_{i-1}\right|-2\left|w_{i}\right|+\left|w_{i+1}\right|\right)+\left(\widetilde{\mathrm{P}}^{\prime}\left(s_{i+1}-\overline{\mathrm{k}}\right)-\widetilde{\mathrm{Q}}\left(s_{i+1}-\overline{\mathrm{k}}\right)\right) \frac{\overline{\mathrm{k}}}{2}\left(\frac{\left(\left|w_{i+1}\right|-\left|w_{i}\right|\right)}{\mathrm{h}}\right) \\
& \quad+\left(\widetilde{\mathrm{P}}^{\prime}\left(s_{i}+\overline{\mathrm{k}}\right)-\widetilde{\mathrm{Q}}\left(s_{i}+\overline{\mathrm{k}}\right)\right)\left(\frac{\bar{k}}{2}\right)\left(\frac{\left(\left|w_{i-1}\right|-\left|w_{i}\right|\right)}{\mathrm{h}}\right)+\left(\widetilde{-\mathrm{P}^{\prime}}\left(s_{i}+\overline{\mathrm{k}}\right)+\widetilde{\mathrm{Q}}\left(s_{i}+\overline{\mathrm{k}}\right)\right)\left(\frac{\mathrm{h}}{2}\right)\left|w_{i}\right| \\
& \quad+\left(\widetilde{-\mathrm{P}^{\prime}}\left(s_{i+1}-\overline{\mathrm{k}}\right)+\widetilde{\mathrm{Q}}\left(s_{i+1}-\overline{\mathrm{k}}\right)\right)\left(\frac{\mathrm{h}}{2}\right)\left|w_{i+1}\right|+\widetilde{\mathrm{P}}^{\prime}\left(s_{i+1}\right) w_{i+1}+\widetilde{\mathrm{P}}^{\prime}\left(s_{i}\right)\left|w_{i}\right| \\
& \quad-\widetilde{\mathrm{Q}}\left(s_{i}\right)\left|w_{i}\right|+\left|-\left\{\frac{\widetilde{\mathrm{F}}\left(s_{i+1}+\overline{\mathrm{k}}\right)+\widetilde{\mathrm{F}}\left(s_{i}+\overline{\mathrm{k}}\right)}{2}\right\}\right| \geqslant 0 .
\end{aligned}
$$

Now in the above inequality (4.2) to replace the coefficients $\varepsilon>0$ and $\mathrm{P}_{\mathrm{i}}$ by same constants, one has to keep in mind the sign of the expressions $\left(\left|w_{i-1}\right|-2\left|w_{i}\right|+\left|w_{i+1}\right|\right),\left(\left|w_{i-1}\right|-\left|w_{i}\right|\right)$, and $\left(\left|w_{i+1}\right|-\left|w_{i}\right|\right)$. If $\left(\left|w_{i-1}\right|-2\left|w_{i}\right|+\left|w_{i+1}\right|\right) \geqslant 0$, we use the inequality $0<(\widetilde{\sigma} \varepsilon)$ and if $\left(\left|w_{i-1}\right|-2\left|w_{i}\right|+\left|w_{i+1}\right|\right) \leqslant 0$, we use the inequality $(\widetilde{\sigma} \varepsilon)<0$. If $\left(\left|w_{i+1}\right|-\left|w_{i}\right|\right) \geqslant 0,\left(\left|w_{i}\right|-\left|w_{i-1}\right|\right) \geqslant 0$, we use the inequality $0<\widetilde{p}\left(s_{i+1}\right) \leqslant$ 
$\left\|\widetilde{p}\left(s_{i+1}\right)\right\|_{h, \infty}$ and if $\left(\left|w_{i+1}\right|-\left|w_{i}\right|\right)<0,\left(\left|w_{i-1}\right|-\left|w_{i}\right|\right)<0$, we use the inequality $\widetilde{\mathrm{P}}\left(s_{i}\right) \geqslant M>0$. Thus using these facts in the above inequality (4.2), we get

$$
C_{1} \frac{\left(\left|w_{i-1}\right|-2\left|w_{i}\right|+\left|w_{i+1}\right|\right)}{h^{2}}+C_{2}\left(\frac{\left(\left|w_{i+1}\right|-\left|w_{i}\right|\right)}{h}\right)-C_{3}\left(\frac{\left(\left|w_{i}\right|-\left|w_{i-1}\right|\right)}{h}\right)+\widetilde{Q}_{i}\left|w_{i}\right|+\left|\widetilde{R}_{i}\right| \geqslant 0,
$$

where $C_{1}, C_{2}, C_{3}$, and $F_{i}$ are positive constants

$$
\begin{aligned}
& \mathrm{C}_{1}=(\widetilde{\sigma} \varepsilon), \\
& C_{2}=\left(\widetilde{\mathrm{P}}^{\prime}\left(s_{i+1}-\overline{\mathrm{k}}\right)-\widetilde{\mathrm{Q}}\left(s_{i+1}-\overline{\mathrm{k}}\right)\right)\left(\frac{\overline{\mathrm{k}}}{2}\right)+\left(\widetilde{-\mathrm{P}}^{\prime}\left(s_{i+1}-\overline{\mathrm{k}}\right)+\widetilde{\mathrm{Q}}\left(s_{i+1}-\overline{\mathrm{k}}\right)\right)\left(\frac{\mathrm{h}}{2}\right)+\widetilde{\mathrm{P}}^{\prime}\left(s_{i}\right), \\
& \mathrm{C}_{3}=\left(\widetilde{\mathrm{P}}^{\prime}\left(s_{i}+\overline{\mathrm{k}}\right)-\widetilde{\mathrm{Q}}\left(s_{i}+\overline{\mathrm{k}}\right)\right)\left(\frac{\overline{\mathrm{k}}}{2}\right)+\left(\widetilde{\widetilde{\mathrm{P}}^{\prime}}\left(s_{i}+\overline{\mathrm{k}}\right)+\widetilde{\mathrm{Q}}\left(s_{i}+\overline{\mathrm{k}}\right)\right)\left(\frac{\mathrm{h}}{2}\right)+\widetilde{\mathrm{P}}^{\prime}\left(s_{i+1}\right), \\
& \widetilde{R}_{i}=-\left\{\frac{\widetilde{F}\left(s_{i+1}+\bar{k}\right)+\widetilde{F}\left(s_{i}+\bar{k}\right)}{2}\right\}
\end{aligned}
$$

are the depending on the sign of the expressions $\left(\left|w_{i-1}\right|-2\left|w_{i}\right|+\left|w_{i+1}\right|\right),\left|w_{i+1}\right|-\left|w_{i}\right|,\left|w_{i-1}\right|-\left|w_{i}\right|$, respectively. Again a rearrangement of the terms in the inequality (4.3) and using the inequality $\left|\widetilde{R}_{\mathfrak{i}}\right| \leqslant$ $\|\widetilde{R}\|_{h, \infty}$ yields

$$
\begin{gathered}
C_{1} \frac{\left(\left|w_{i+1}\right|-\left|w_{i}\right|\right)}{h^{2}}-C_{1} \frac{\left(\left|w_{i}\right|-\left|w_{i-1}\right|\right)}{h^{2}}+C_{2}\left(\frac{\left(\left|w_{i+1}\right|-\left|w_{i}\right|\right)}{h}\right)-C_{3}\left(\frac{\left(\left|w_{i}\right|-\left|w_{i-1}\right|\right)}{h}\right) \\
+\widetilde{Q}_{i}\left|w_{i}\right|+\|\widetilde{R}\|_{h, \infty} \geqslant 0 .
\end{gathered}
$$

To prove the uniqueness and exitance, let $\left(u_{i}\right)_{i=0}^{N},\left(v_{i}\right)_{i=0}^{N}$ be two sets of solutions of the difference equation (2.11) satisfying the boundary conditions. Suppose $w_{i}=u_{i}-v_{i}$, then $w_{i}$ satisfies

$$
\mathrm{E}_{1}^{\mathrm{N}} \mathrm{w}_{\mathrm{i}}=\widetilde{\mathrm{R}}_{\mathrm{i}}
$$

where $\widetilde{R}_{i}=0, \forall i, 1 \leqslant i \leqslant N-1$, and $w_{0}=0=w_{N}$. Summing (4.5) over $i=1,2,3, \ldots, N-1$, we obtain

$$
C_{1} \frac{\left(\left|w_{i+1}\right|-\left|w_{i}\right|\right)}{h^{2}}-C_{1} \frac{\left(\left|w_{i}\right|-\left|w_{i-1}\right|\right)}{h^{2}}+C_{2}\left(\frac{\left(\left|w_{i+1}\right|-\left|w_{i}\right|\right)}{h}\right)-C_{3} \frac{\left(\left|w_{i+1}\right|-\left|w_{i}\right|\right)}{h^{2}}+\sum_{i=1}^{N-1} \widetilde{Q}_{i}\left|w_{i}\right| \geqslant 0
$$

Since $C_{1}>0, C_{2}>0, C_{3}>0$, and $\left|w_{i}\right| \geqslant 0, \forall i, i=1,2,3, \ldots, N-1$, therefore for the inequality (4.5) holds, we must have $w_{i}=0, \forall, i=1,2,3, \ldots, N-1$. This implies the uniqueness of the solution to the tridiagonal system of difference equation (2.11). For linear equations, the existance is implied by uniqueness. Now to obtain the required bounds on the solution of the discrete problem $\left(w_{i}\right)_{i=0}^{N}$, set $y_{i}=w_{i}+l_{i}$, where $y_{i}$ satisfies the difference equation (2.11) the boundary condition $l_{i}=(1-i h) \phi_{0}+(i h) \beta$. Then $y_{0}=0=y_{N}$, and $y_{i}, i=1,2,3, \ldots, N-1$, satisfies $L_{1}^{N} y_{i}=\widetilde{R}_{i}$. Now let

$$
\left|y_{n}\right|=\|y\|_{h, \infty} \geqslant\left|y_{i}\right|, \quad \forall i=1,2,3, \ldots, N .
$$

Then summing (4.4) from $i=n$ to $N-1$ and using the inequality $P(s) \geqslant M>0$ and $0<\widetilde{P}\left(s_{i+1}\right) \leqslant$ $\left\|\mathrm{P}\left(s_{i+1}\right)\right\|_{h, \infty}>0$ gives

$$
-C_{1} \frac{\left(\left|y_{n}\right|-\left|y_{N-1}\right|\right)}{h^{2}}-\widetilde{C_{1}} \frac{\left(\left|y_{N-1}\right|\right)}{h^{2}}+C_{2} \frac{\left|y_{n}\right|}{h}+\sum_{i=1}^{N-1} \widetilde{Q}_{i}\left|y_{i}\right|+(N-n-1)\|\widetilde{R}\|_{h, \infty} \geqslant 0
$$


Since $Q(s) \leqslant 0$ and from inequality (4.6), we gave $\left(\left|y_{n+1}\right|-\left|y_{n}\right|\right) \geqslant 0,\left(\left|y_{n}\right|-\left|y_{n-1}\right|\right) \geqslant 0$, therefore the first, second, third and fourth terms on the left sides in the inequality (4.7) are negative, so after omitting these terms from the inequality, it reduces to $C_{2}\left|y_{n}\right| \leqslant(N-n-1) h\|\widetilde{R}\|_{h, \infty} \leqslant\|\widetilde{R}\|_{h, \infty}$, since $(N-n-$ $1) h \leqslant 1$. We have

$$
\left|y_{n}\right| \leqslant C_{2}^{-1}|| \widetilde{R} \|_{h, \infty}
$$

Also, we have $w_{i}=y_{i}+l_{i}$

$$
\|w\|_{h, \infty}=\max _{0 \leqslant i \leqslant N}\left|w_{i}\right| \leqslant\|y\|_{h, \infty}+\|l\|_{h, \infty}\|w\|_{h, \infty} \leqslant\left|y_{h}\right|+\|l\|_{h, \infty} .
$$

Now to complete the estimate, we have to find out the bound on $l_{i}$,

$$
\|l\|_{h, \infty}=\max _{0 \leqslant i \leqslant N}\left|l_{i}\right| \leqslant \max _{0 \leqslant i \leqslant N}\left[(1-i h) \phi_{0}+(i h) \beta\right] .
$$

We have

$$
\|l\|_{h, \infty} \leqslant\left|\phi_{0}\right|+|\beta| \leqslant\|\phi\|_{h, \infty}+|\beta| .
$$

From Eqs. (4.8)-(4.10), we obtain the required estimate. Therefore,

$$
\|w\|_{h, \infty} \leqslant C^{-1}\left|\widetilde{R}\left\|_{h, \infty}+\right\| \phi \|_{h, \infty}+\right| \beta \mid,
$$

where $C=M$ or $\|P\|_{h, \infty}$. Here $\|.\|_{h, \infty}$ is the discrete $l_{\infty}$-norm, given by $\|s\|_{h, \infty}=\max _{0 \leqslant i \leqslant N}\left|s_{i}\right|$.

This theorem implies that the solution to the system of the difference equation (2.11) is uniformly bounded, independent of the mesh size $h$ and the singular perturbed parameter $\varepsilon$. Thus the left end scheme is stable for all step size. One can analysis of remaining three cases.

\section{Convergence analysis}

Incorporating the boundary conditions, the matrix form of the system of equations is

$$
(\mathrm{X}+\mathrm{Y}) w+\mathrm{U}+\tau(\mathrm{h})=0
$$
where $X=\left[\frac{\widetilde{\sigma} \varepsilon}{h}, \frac{-2 \widetilde{\sigma} \varepsilon}{h}, \frac{\widetilde{\sigma} \varepsilon}{h}\right]=\left[\begin{array}{cccccc}\frac{-2 \widetilde{\sigma} \varepsilon}{h} & \frac{\widetilde{\sigma} \varepsilon}{h} & 0 & 0 & \ldots & 0 \\ \frac{\sigma \varepsilon}{h} & \frac{-2 \tilde{\sigma} \varepsilon}{h} & \frac{\widetilde{\sigma} \varepsilon}{h} & 0 & \ldots & 0 \\ 0 & \frac{\widetilde{\sigma} \varepsilon}{h} & \frac{-2 \tilde{\sigma} \varepsilon}{h} & \frac{\tilde{\sigma} \varepsilon}{h} & \ldots & 0 \\ \ldots & \ldots & \ldots & \ldots & \ldots & \ldots \\ \ldots & \ldots & \ldots & \ldots & \ldots & \ldots \\ 0 & \ldots & \ldots & 0 & \frac{\tilde{\sigma} \varepsilon}{h} & \frac{-2 \tilde{\sigma} \varepsilon}{h}\end{array}\right]$,
and

$$
\tilde{Y}=\left[\tilde{z}_{i}, \tilde{v}_{i}, \tilde{w}_{i}\right]=\left[\begin{array}{cccccc}
\tilde{v}_{1} & \tilde{w}_{1} & 0 & 0 & \ldots & 0 \\
\tilde{z}_{2} & \tilde{v}_{2} & \tilde{w}_{2} & 0 & \ldots & 0 \\
0 & \tilde{z}_{3} & \tilde{v}_{3} & \tilde{w}_{3} & \ldots & 0 \\
\ldots & \ldots & \ldots & \ldots & \ldots & \ldots \\
\ldots & \ldots & \ldots & \ldots & \ldots & \ldots \\
0 & \ldots & \ldots & 0 & \tilde{z}_{N-1} & \tilde{v}_{N-1}
\end{array}\right]
$$

where

$$
\begin{aligned}
& \widetilde{z}_{i}=\frac{\widetilde{k}}{2}\left(\widetilde{P}\left(s_{i}+\widetilde{k}\right)-\widetilde{Q}\left(s_{i}+\widetilde{k}\right)\right), \\
& \widetilde{v}_{i}=\left\{-\widetilde{P}\left(s_{i}\right)-\widetilde{P}^{\prime}\left(s_{i+1}-\widetilde{k}\right) \frac{\widetilde{k}}{2}-\widetilde{P}^{\prime}\left(s_{i}+\widetilde{k}\right)\left(\frac{h+\widetilde{k}}{2}\right)+\widetilde{Q}\left(s_{i+1}-\widetilde{k}\right) \frac{\widetilde{k}}{2}+\widetilde{Q}\left(s_{i}+\widetilde{k}\right)\left(\frac{h+\widetilde{k}}{2}\right)\right\},
\end{aligned}
$$




$$
\begin{aligned}
\widetilde{w}_{i} & =\left\{\widetilde{P}(s)-\widetilde{P}^{\prime}\left(s_{i+1}-\widetilde{k}\right)\left(\frac{h+\widetilde{k}}{2}\right)+\widetilde{Q}\left(s_{i+1}-\widetilde{k}\right)\left(\frac{h-\widetilde{k}}{2}\right)\right\}, \\
\widetilde{Q} & =\left\{a_{1}+\left(\frac{\varepsilon}{h}+z_{1}\right) \gamma_{0}, a_{2}, a_{3, \ldots,} a_{N-2,} a_{N-1}+\left(\frac{\varepsilon}{h}+\widetilde{w}_{N-1}\right) \gamma_{1}\right\}, \\
a_{i} & =\frac{h}{2}\left\{\widetilde{F}\left(\theta_{i+1}-\widetilde{k}\right)+\widetilde{F}\left(s_{i}+\widetilde{k}\right)\right\},
\end{aligned}
$$

where $i=1,2, \ldots, N-1$. The local truncation error with the proposed scheme is

$$
\tilde{\tau}(h)=-\frac{h}{2}\left(\tilde{P^{\prime}}\left(s_{i+1}-\tilde{K}\right)+\tilde{P^{\prime}}\left(s_{i}+\tilde{K}\right)\right) w_{i}+O\left(h^{2}\right) .
$$

Let $w=\left[w_{1}, w_{2}, \ldots, w_{N-1}\right]^{\top}, \tau(h)=\left[s_{1}, s_{2}, \ldots, s_{N-1}\right]^{\top}, \widetilde{\mathrm{O}}=[0,0, \ldots, 0]^{\top}$ are associated vector of Eq. (5.1). Let $W=\left[w_{1}, w_{2}, \ldots, w_{N-1}\right]^{\top} \cong \widetilde{W}$ satisfies the equation

$$
(\mathrm{X}+\mathrm{Y}) \mathrm{W}+\mathrm{U}=0
$$

Let $l_{i}=w_{i}-\widetilde{W}_{i}, \forall i=1,2, \ldots, N-1$ be the discretization error $L=\left[l_{1}, l_{2}, \ldots, l_{N-1}\right]^{\top}=w-\widetilde{W}$. So that Subtract (5.1)-(5.2), then the error equation is

$$
(X+Y) L=\tau(h) .
$$

Let $|\widetilde{P}(t)| \leqslant \widetilde{c}_{1}$ and $|\widetilde{Q}(t)| \leqslant \widetilde{c}_{2}$, where $\widetilde{c}_{1}, \widetilde{c}_{2}$ is a positive constant if be the $[i, j]$ th element of $\tilde{M}$, then $\left|\widetilde{P}_{i, i+1}\right|=\left|\widetilde{w}_{i}\right| \leqslant\left(\widetilde{p}\left(s_{i+1}\right)+\widetilde{P}^{\prime}\left(s_{i+1}-\widetilde{k}\right)\left(\frac{h+\widetilde{k}}{2}\right)+\widetilde{Q}\left(s_{i+1}-\widetilde{k}\right)\left(\frac{h-\widetilde{k}}{2}\right)\right) \widetilde{c}_{1}$, where $i=1,2, \ldots, N-2$, $\left|\widetilde{P}_{i-1}\right|=\left|\widetilde{z}_{i}\right| \leqslant \frac{\widetilde{k}}{2}\left[\widetilde{P}^{\prime}\left(s_{i}+\widetilde{k}\right)-\widetilde{Q}\left(s_{i}+\widetilde{k}\right)\right] \widetilde{c}_{2}$, where $i=1,2, \ldots, N-1$. Hence for sufficiently small $h$, we have

$$
\left|\widetilde{p}_{i, i+1}\right|<\varepsilon \text { for } i=1,2, \ldots, N-2,
$$

and

$$
\left|\widetilde{\mathrm{P}}_{i, i-1}\right|<\varepsilon \text { for } i=2,3, \ldots, N-1 \text {. }
$$

Hence, $(X+Y)$ is irreducible.

Let $\tilde{S}_{i}$ the sum of the elements of the ith row of the matrix $(X+Y)$ be $\tilde{\theta}_{i}$, then we have

$$
\begin{aligned}
\widetilde{S}_{1}=\sum_{j=1}^{N-1} \widetilde{M}_{i j}= & \left\{\widetilde{P}\left(s_{i+1}\right)-\widetilde{P}\left(s_{i}\right)+\frac{\widetilde{\sigma} \varepsilon}{h}-\widetilde{P}^{\prime}\left(s_{i}+\widetilde{k}\right) h_{1}+\widetilde{Q}\left(s_{i}+\widetilde{k}\right) h_{1}\right\} \\
& +\left\{\frac{h}{2}\left(\widetilde{P}\left(s_{i+1}-\widetilde{k}\right)-\widetilde{P}^{\prime}\left(s_{i+1}-\widetilde{k}\right)\right)\right\}
\end{aligned}
$$

for $i=1$, where $h_{1}=\left(\frac{h+\widetilde{k}}{2}\right)$,

$$
\tilde{S}_{N-1}=\sum_{j=1}^{N-1} \tilde{m}_{N-1 j}=-\tilde{P}\left(\theta_{i}\right)-\frac{\varepsilon}{h}-\tilde{P^{\prime}}\left(s_{i+1}-\tilde{K}\right) \frac{h}{2}-\tilde{P^{\prime}}\left(s_{i}+\tilde{K}\right) \frac{\tilde{K}}{2}+\tilde{Q}\left(s_{i+1}-\tilde{K}\right) \frac{\tilde{K}}{2}+\tilde{Q}\left(s_{i}+\tilde{K}\right) \frac{h}{2},
$$

for $i=2,3, \ldots, N-2$,

$$
\tilde{S}_{N-1}=\sum_{j=1}^{N-1} \widetilde{M}_{N-1 j}=-\widetilde{P}\left(s_{i}\right)-\frac{\widetilde{\sigma} \varepsilon}{h}-\widetilde{P}^{\prime}\left(s_{i+1}-\widetilde{k}\right) \frac{h}{2}-\widetilde{P}^{\prime}\left(s_{i}+\widetilde{k}\right) \frac{\widetilde{k}}{2}+\widetilde{Q}\left(s_{i+1}-\widetilde{k}\right) \frac{\widetilde{k}}{2}+\widetilde{Q}\left(s_{i}+\widetilde{k}\right) \frac{h}{2}
$$


for $i=N-1$. Let $\widetilde{C}_{i}=\min |\widetilde{p}(s)|$ and $\widetilde{C}_{1}^{*}=\max |\widetilde{p}(s)|$ since $0<\varepsilon \leqslant 1$ and its verify that for sufficiently small $h,(X+Y)$, is monotonic. Hence $(X+Y)^{-1}$ exits and $(X+Y)^{-1} \geqslant 0$, thus by Eq. (5.3) we get

$$
\|\mathrm{L}\| \leqslant\left\|(\tilde{\mathrm{X}}+\tilde{\mathrm{Y}})^{-1}\right\|\|\tilde{\tau}\|
$$

For sufficiently small $h$, we have $\widetilde{s}_{i}>\left(\widetilde{P}\left(s_{i+1}\right)-\widetilde{P}\left(s_{i}\right)\right) \Rightarrow \bar{s}_{1}>$ constant, $\widetilde{s}_{i}>\left(\widetilde{P}\left(s_{i+1}\right)-\widetilde{P}\left(s_{i}\right)\right)$ for $i=N-1 \Rightarrow \bar{s}_{i}>$ constant, $\widetilde{s}_{i}>\widetilde{P}\left(s_{i}\right)$ for $i=2,3, \ldots, N-2$. Let $(X+Y)_{(i, k)}^{-1}$ be the $(i, k)$ th elements of $(\mathrm{X}+\mathrm{Y})^{-1}$ and we define

$$
\left\|(X+Y)^{-1}\right\|=\max \sum_{k=1}^{N-1}(X+Y)_{(i k)^{\prime}}^{-1}
$$

and

$$
\|\tilde{\tau}(h)\|=\max |\tilde{\tau}(h)|
$$

Since $(X+Y)_{(i k)}^{-1} \geqslant 0$ and $\sum_{k=1}^{N-1}(X+Y)_{(i k)}^{-1} s_{k}=1$ for $i=1,2, \ldots, N-1$, we have

$$
\begin{gathered}
(X+Y)_{(i k)}^{-1} \leqslant \frac{1}{s_{k}} \leqslant \frac{1}{C^{\prime}} \\
(X+Y)_{(i N-1)}^{-1} \leqslant \frac{1}{s_{N-1}} \leqslant \frac{1}{C} .
\end{gathered}
$$

Furthermore,

$$
\sum_{k=2}^{N-2}(X+Y)_{(i k)}^{-1} \leqslant \frac{1}{\min s_{k}} \leqslant \frac{1}{C^{\prime}}, \forall i=1,2,3, \ldots, N-1 .
$$

With the use of (5.5)-(5.7), from Eq. (5.4), we have $\|\mathrm{L}\| \leqslant \mathrm{O}(\mathrm{h})$. Hence, first order convergent is achieved for the suggested scheme. In similar way, the convergence in other cases can be analyzed.

\section{Numerical experiments}

To describe the proposed scheme computationally, it is implemented on left end layer, right end layer, dual layer and internal layer problems. These problems are discussed in the literature and numerical results are available for comparison. The maximum absolute error $L_{\varepsilon}^{N}=\max _{0 \leqslant i \leqslant N}\left|w\left(s_{i}\right)-w_{i}\right|$ is calculated for each example with comparison. Here $w\left(s_{i}\right)$ is the exact solution and $w_{i}$ is the numerical solution. We use double mesh principle [3], $L_{\varepsilon}^{N}=\max _{0 \leqslant i \leqslant N}\left|w_{i}^{N}-w_{2 i}^{2 N}\right|$ to find the maximum errors for the examples, where exact solution is not known. Here $w_{i}^{N}$ is the numerical solution with $N$ subintervals and $w_{2 i}^{2 N}$ is the numerical solution with $2 \mathrm{~N}$ subintervals.

Example 6.1. $\varepsilon w^{\prime \prime}(\mathrm{s})+w^{\prime}(s-\delta)-w(s)=0$ with $w(0)=1$ and $w(1)=1$. The exact solution is: $w(s)=\frac{\left(\left(1-e^{c_{2}}\right) e^{c_{1} s}+\left(e^{c_{1}}-1\right) e^{c_{2} s}\right)}{\left(e^{c_{1}}-e^{c_{2}}\right)}$, where

$$
c_{1}=\frac{(-1-\sqrt{(1+4(\varepsilon-\delta)))}}{2(\varepsilon-\delta)}, \quad c_{2}=\frac{(-1+\sqrt{(1+4(\varepsilon-\delta)))}}{2(\varepsilon-\delta)} .
$$

Example 6.2. $\varepsilon w^{\prime \prime}(s)+e^{-0.5 s} w^{\prime}(s-\delta)-w(s)=0$ with $w(0)=1, w(1)=1$. For this problem exact solution is not known. 
Example 6.3. $\varepsilon w^{\prime \prime}(\mathrm{s})-w^{\prime}(s-\delta)-w(s)=0$ with $w(0)=1$ and $w(1)=-1$. The exact solution is: $w(s)=\frac{\left(\left(1+e^{c_{2}}\right) e^{c_{1} s}-\left(e^{c_{1}}+1\right) e^{c_{2} s}\right)}{\left(e^{c_{2}}-e^{c_{1}}\right)}$, where

$$
c_{1}=\frac{(-1-\sqrt{(1+4(\varepsilon+\delta)))}}{2(\varepsilon+\delta)}, \quad c_{2}=\frac{(-1+\sqrt{(1+4(\varepsilon+\delta)))}}{2(\varepsilon+\delta)} .
$$

Example 6.4. $\varepsilon w^{\prime \prime}(s)-e^{t} w^{\prime}(s-\delta)-t w(s)=0$ with $w(0)=1, w(1)=1$. For this problem exact solution is not known.

Example 6.5. Consider the dual layer problem $\varepsilon w^{\prime \prime}(s)+2(1-2 t) w^{\prime}(s-\delta)+4 w(s)=0$, with $w(0)=1$ on $-\delta \leqslant s \leqslant 0, w(1)=1$. For this problem exact solution is not known.

Example 6.6. Consider the dual layer problem $\varepsilon w^{\prime \prime}(s)+2(1-2 t) w^{\prime}(s-\delta)+4 w(s)=4(1-4 s)$, with $w(s)=1$ on $-\delta \leqslant s \leqslant 0, w(1)=1$. For this problem exact solution is not available.

Example 6.7. Consider an internal layer problem $\varepsilon w^{\prime \prime}(s)+2(2 s-1) w^{\prime}(s-\delta)-4 w(s)=0$, with $w(s)=$ 1 on $-\delta \leqslant s \leqslant 0, w(1)=1$. Exact solution is not known for this problem.

Example 6.8. Consider the internal layer problem $\varepsilon w^{\prime \prime}(s)+2(2 s-1) w^{\prime}(s-\delta)-4 w(s)=4(4 s-1)$, with $w(s)=1$ on $-\delta \leqslant \theta \leqslant 0, w(1)=1$. Exact solution is not available for this problem.

\section{Discussions and conclusion}

A quadrature approach for solving a differential equations having a delay and layer structure is suggested. By taking the perturbation parameter into the differentiated term, the given problem is reduced into first order neutral type differential equation. Then Gaussian quadrature involving fitting parameter is implemented to get the difference scheme for the problem. Thomas algorithm is employed to solve this difference scheme. The suggested technique is applied on several test problems to explain the method and consequences of the delay in the layer shown in Figures 1-8.

Using the Figures 1-6 presented in this paper, we found that as the delay $\delta$ increases, the thickness of the left-end boundary layer decreases, and the thickness of right-end boundary layer increases as $\delta$ increases. From Figures 7 and 8 , it is observed that, as the $\delta$ increases the width of the internal layer decreases. Tables 1-8, list the numerical results to support the predicted theory for the examples considered. From the results it is noticed that when mesh size h decreases, the maximum error decreases, which shows the computed solution convergence. The numerical scheme is analyzed for convergence. The suggested method is easy to implement and gives good results to the proposed problem.

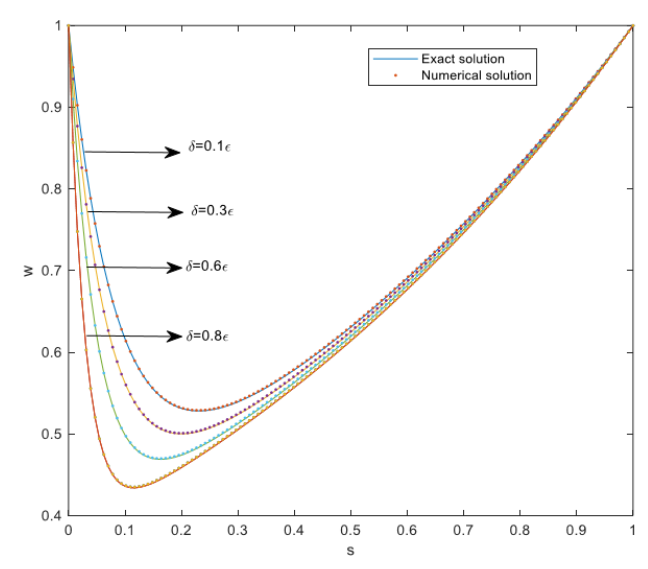

Figure 1: Numerical solution of Example 6.1 with $\varepsilon=0.01 . \quad$ Figure 2: Numerical solution of Example 6.2 with $\varepsilon=0.1$.

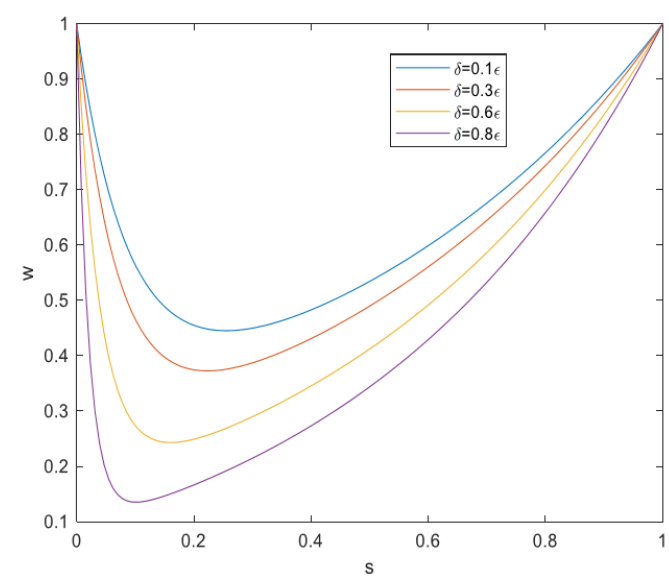



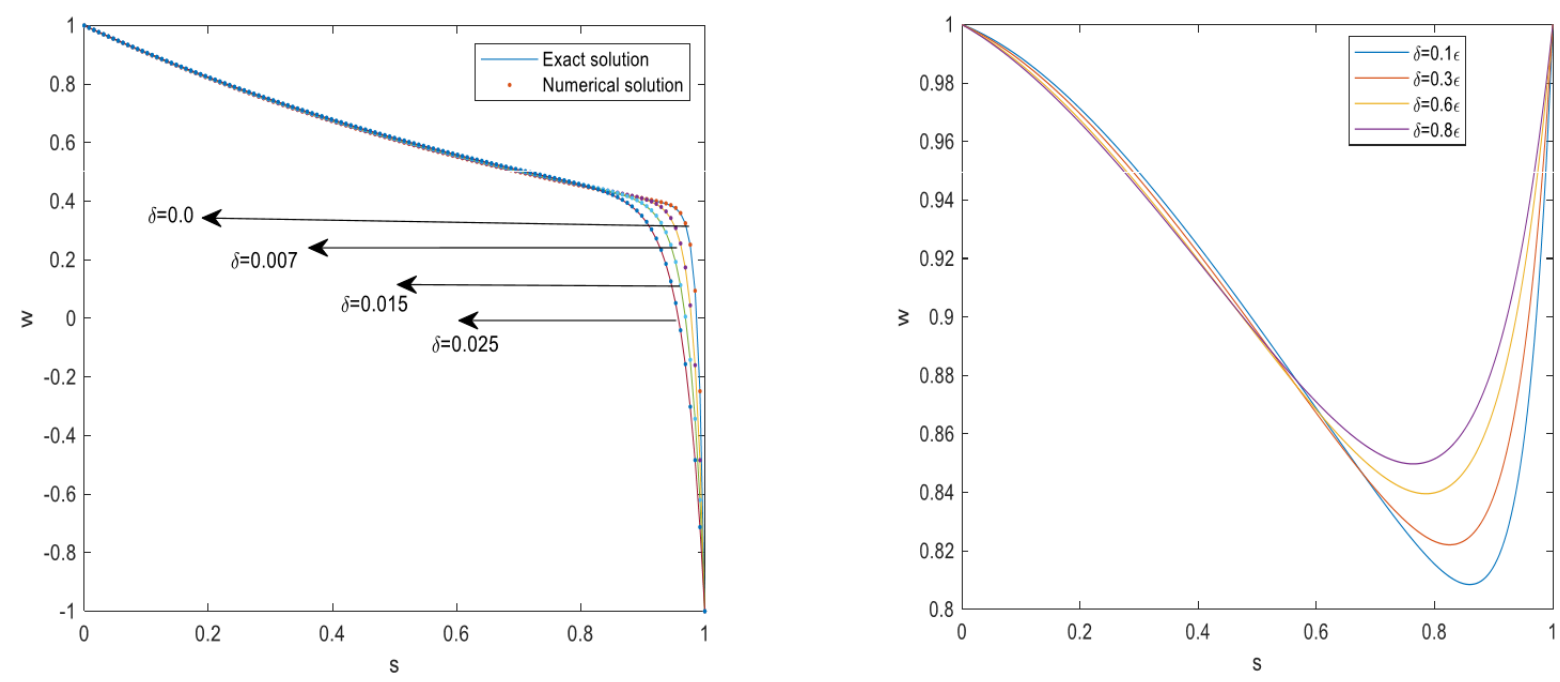

Figure 3: Numerical solution of Example 6.3 with $\varepsilon=0.01 . \quad$ Figure 4: Numerical solution of Example 6.4 with $\varepsilon=0.1$.
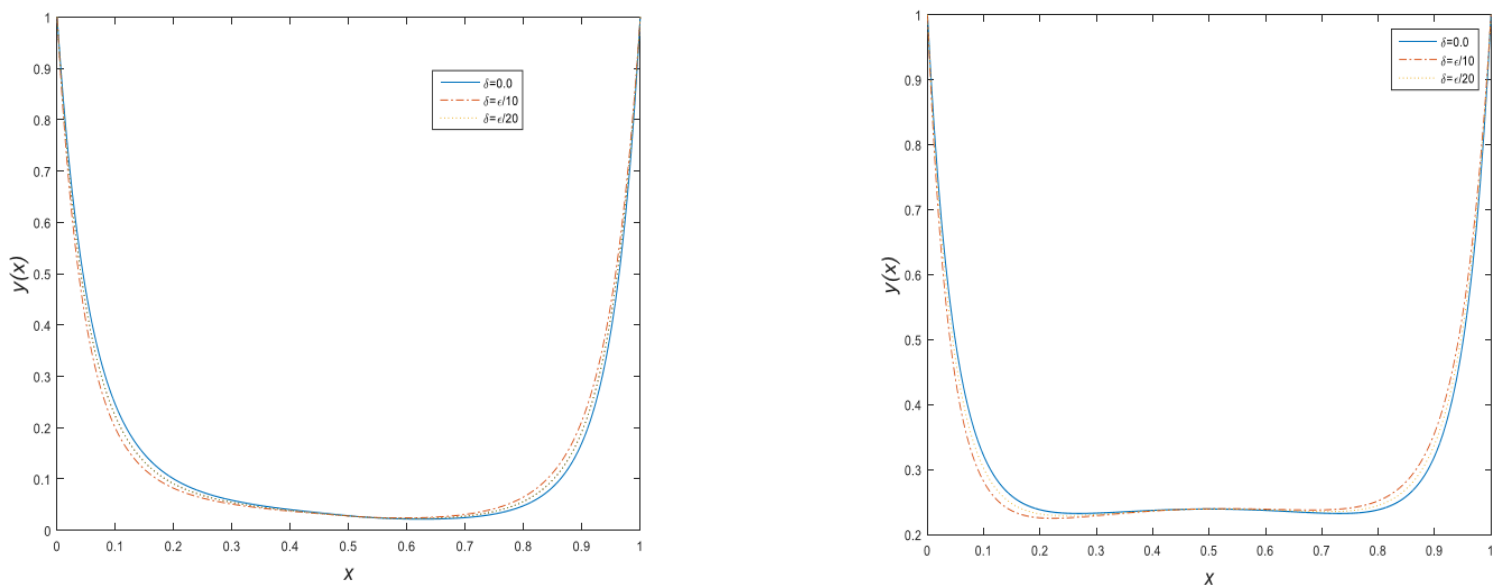

Figure 5: Numerical solution of Example 6.5 with $\varepsilon=0.1$.

Figure 6: Numerical solution of Example 6.6 with $\varepsilon=0.1$.
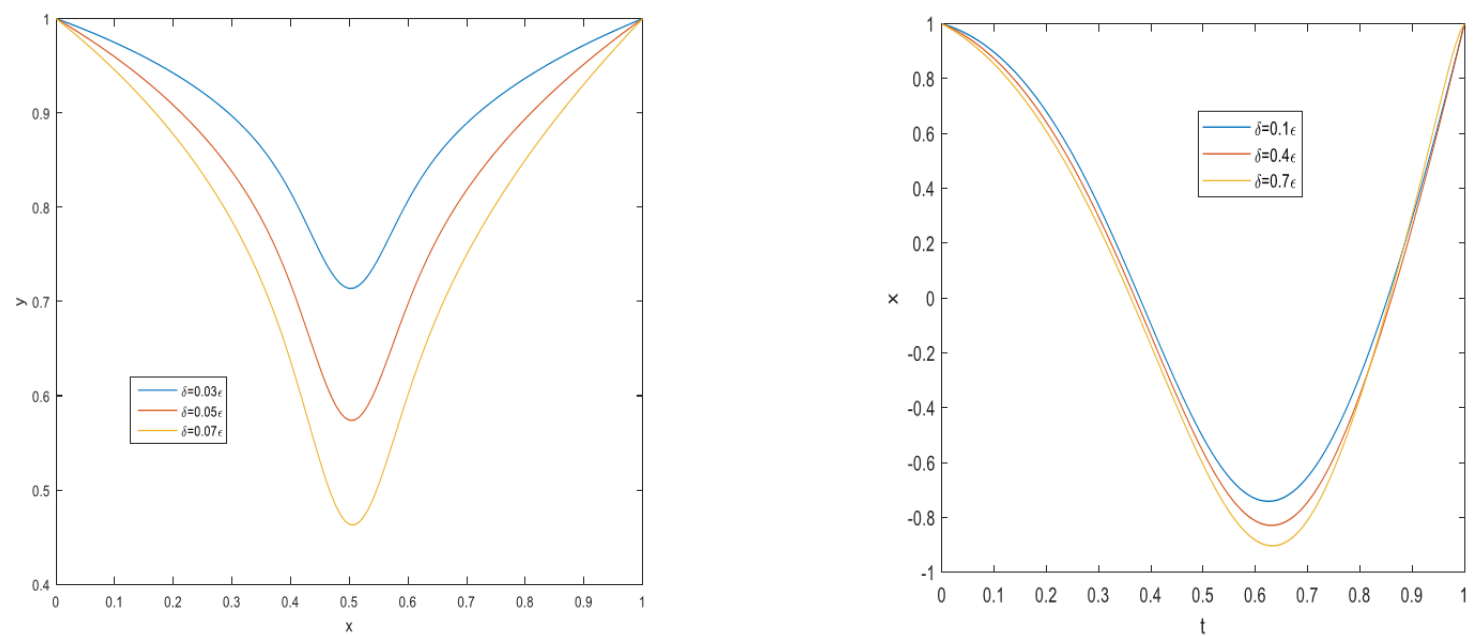

Figure 7: Numerical solution of Example 6.7 with $\varepsilon=0.1 . \quad$ Figure 8: Numerical solution of Example 6.8 with $\varepsilon=0.1$. 
Table 1: Maximum errors of Example 6.1 for $\varepsilon=0.01$ and for different values of $\delta$.

\begin{tabular}{ccccc}
\hline$\delta \downarrow$ & $\mathrm{N}=10^{2}$ & $\mathrm{~N}=10^{3}$ & $\mathrm{~N}=10^{4}$ & $\mathrm{~N}=10^{5}$ \\
\hline \multicolumn{5}{c}{ Present method } \\
0.001 & $1.4744(-3)$ & $1.7640(-4)$ & $1.7933(-5)$ & $1.7922(-6)$ \\
0.003 & $1.3940(-3)$ & $1.7649(-4)$ & $1.8030(-5)$ & $1.8071(-6)$ \\
0.006 & $1.1333(-3)$ & $1.7469(-4)$ & $1.8146(-5)$ & $1.8219(-6)$ \\
0.008 & $7.0748(-4)$ & $1.6798(-4)$ & $1.8161(-5)$ & $1.8304(-6)$ \\
5 & \multicolumn{4}{c}{ Results in $[6]$} \\
0.001 & $9.0928(-2)$ & $1.2290(-2)$ & $1.2794(-3)$ & $1.2847(-4)$ \\
0.003 & $1.08362(-1)$ & $1.5626(-2)$ & $1.6446(-3)$ & $1.6534(-4)$ \\
0.006 & $1.28454(-1)$ & $2.6314(-2)$ & $2.8703(-3)$ & $2.8971(-4)$ \\
0.008 & $1.01499(-1)$ & $4.8347(-2)$ & $5.6889(-3)$ & $5.7948(-4)$ \\
\hline
\end{tabular}

Table 2: Maximum errors of Example 6.2 for $\varepsilon=0.1$ and for different values of $\delta$.

\begin{tabular}{cccc}
\hline$\delta \downarrow$ & $\mathrm{N}=10^{2}$ & $\mathrm{~N}=10^{3}$ & $\mathrm{~N}=10^{4}$ \\
\hline \multicolumn{4}{c}{ Present method } \\
0.01 & $3.0808(-3)$ & $3.1574(-4)$ & $3.1644(-5)$ \\
0.03 & $3.7714(-3)$ & $3.8943(-4)$ & $3.9054(-5)$ \\
0.06 & $5.4990(-3)$ & $5.8129(-4)$ & $5.8399(-5)$ \\
0.08 & $8.5969(-3)$ & $9.7844(-4)$ & $9.8768(-5)$ \\
\multicolumn{4}{c}{ Results in $[12]$} \\
0.01 & $6.3299(-3)$ & $6.7426(-4)$ & $6.7871(-5)$ \\
0.03 & $8.1591(-3)$ & $8.8256(-4)$ & $8.8986(-5)$ \\
0.06 & $1.3847(-2)$ & $1.5797(-3)$ & $1.6020(-4)$ \\
0.08 & $2.4771(-2)$ & $3.1732(-3)$ & $3.2602(-4)$ \\
\hline
\end{tabular}

Table 3: Maximum errors of Example 6.3 for $\varepsilon=0.01$ and for different values of $\delta$.

\begin{tabular}{ccccc}
\hline$\delta \downarrow$ & $\mathrm{N}=10^{2}$ & $\mathrm{~N}=10^{3}$ & $\mathrm{~N}=10^{4}$ & $\mathrm{~N}=10^{5}$ \\
\hline \multicolumn{5}{c}{ Present method } \\
0.00 & $3.0958(-3)$ & $3.6072(-4)$ & $3.6860(-5)$ & $3.6928(-6)$ \\
0.007 & $3.0636(-3)$ & $3.6079(-4)$ & $3.6820(-5)$ & $3.6862(-6)$ \\
0.015 & $3.0388(-3)$ & $3.6085(-4)$ & $3.6774(-5)$ & $3.6889(-6)$ \\
0.025 & $3.1182(-3)$ & $3.6089(-4)$ & $3.6715(-5)$ & $3.6727(-6)$ \\
\multicolumn{5}{c}{ Results in [6] } \\
0.00 & $1.7855(-1)$ & $2.3879(-2)$ & $2.4764(-3)$ & $2.4857(-4)$ \\
0.007 & $1.1763(-1)$ & $1.3951(-2)$ & $1.4225(-3)$ & $1.4254(-4)$ \\
0.015 & $8.3515(-2)$ & $9.4403(-3)$ & $9.5984(-4)$ & $9.6155(-5)$ \\
0.025 & $6.1475(-2)$ & $6.7886(-3)$ & $6.8675(-4)$ & $6.8761(-5)$ \\
\hline
\end{tabular}

Table 4: Maximum errors of Example 6.4 for $\varepsilon=0.1$ and for different values of $\delta$.

\begin{tabular}{cccc}
\hline$\delta \downarrow$ & $\mathrm{N}=10^{2}$ & $\mathrm{~N}=10^{3}$ & $\mathrm{~N}=10^{4}$ \\
\hline \multicolumn{4}{c}{ Present method } \\
0.01 & $4.0262(-3)$ & $4.9360(-4)$ & $4.0316(-5)$ \\
0.03 & $2.7251(-3)$ & $2.0700(-4)$ & $3.1061(-5)$ \\
0.06 & $2.1520(-3)$ & $2.3832(-4)$ & $2.3962(-5)$ \\
0.08 & $1.7733(-3)$ & $1.8480(-4)$ & $1.8557(-5)$ \\
\multicolumn{4}{c}{ Results in [12] } \\
0.01 & $5.7597(-3)$ & $5.0842(-4)$ & $5.0247(-5)$ \\
0.03 & $3.9327(-3)$ & $3.6132(-4)$ & $3.5838(-5)$ \\
0.06 & $2.7025(-3)$ & $2.5507(-4)$ & $2.5364(-5)$ \\
0.08 & $2.2468(-3)$ & $2.1413(-4)$ & $2.1313(-5)$ \\
\hline
\end{tabular}


Table 5: The maximum absolute errors of Example 6.5 for $\delta=\varepsilon / 10$.

\begin{tabular}{lllllll}
\hline$\varepsilon \backslash \hbar$ & $h=2^{-4}$ & $h=2^{-6}$ & $h=2^{-7}$ & $h=2^{-8}$ & $h=2^{-9}$ & $h=2^{-10}$ \\
\hline $2^{-4}$ & $3.181(-2)$ & $1.706(-2)$ & $8.698(-3)$ & $4.381(-3)$ & $2.198(-3)$ & $1.101(-3)$ \\
$2^{-6}$ & $3.150(-2)$ & $2.501(-2)$ & $1.322(-2)$ & $6.705(-3)$ & $3.355(-3)$ & $1.675(-3)$ \\
$2^{-8}$ & $2.968(-2)$ & $2.282(-2)$ & $1.205(-2)$ & $6.013(-3)$ & $5.270(-3)$ & $2.829(-3)$ \\
$2^{-10}$ & $3.793(-2)$ & $2.164(-2)$ & $1.440(-2)$ & $8.785(-3)$ & $4.994(-3)$ & $4.727(-3)$ \\
$2^{-12}$ & $3.444(-2)$ & $2.729(-2)$ & $1.405(-2)$ & $7.656(-3)$ & $4.535(-3)$ & $4.120(-3)$ \\
$2^{-14}$ & $3.273(-2)$ & $2.474(-2)$ & $1.398(-2)$ & $7.573(-3)$ & $4.228(-3)$ & $2.304(-3)$ \\
$2^{-16}$ & $3.223(-2)$ & $2.418(-2)$ & $1.396(-2)$ & $7.557(-3)$ & $3.935(-3)$ & $2.582(-3)$ \\
$2^{-18}$ & $3.210(-2)$ & $2.417(-2)$ & $1.396(-2)$ & $7.552(-3)$ & $3.931(-3)$ & $2.002(-3)$ \\
$2^{-20}$ & $3.207(-2)$ & $2.417(-2)$ & $1.396(-2)$ & $7.551(-3)$ & $3.930(-3)$ & $2.002(-3)$ \\
& & & Results in $[10]$ & & \\
$2^{-4}$ & $3.618(-2)$ & $2.334(-2)$ & $1.447(-2)$ & $8.597(-3)$ & $4.960(-3)$ & $2.797(-3)$ \\
$2^{-6}$ & $3.950(-2)$ & $2.575(-2)$ & $1.579(-2)$ & $9.291(-3)$ & $5.327(-3)$ & $2.996(-3)$ \\
$2^{-8}$ & $3.970(-2)$ & $2.602(-2)$ & $1.606(-2)$ & $9.481(-3)$ & $5.439(-3)$ & $3.054(-3)$ \\
$2^{-10}$ & $3.963(-2)$ & $2.594(-2)$ & $1.602(-2)$ & $9.473(-3)$ & $5.451(-3)$ & $3.069(-3)$ \\
$2^{-12}$ & $3.960(-2)$ & $2.590(-2)$ & $1.598(-2)$ & $9.449(-3)$ & $5.437(-3)$ & $3.063(-3)$ \\
$2^{-14}$ & $3.959(-2)$ & $2.589(-2)$ & $1.597(-2)$ & $9.441(-3)$ & $5.430(-3)$ & $3.058(-3)$ \\
$2^{-16}$ & $3.959(-2)$ & $2.589(-2)$ & $1.597(-2)$ & $9.438(-3)$ & $5.428(-3)$ & $3.056(-3)$ \\
$2^{-18}$ & $3.959(-2)$ & $2.589(-2)$ & $1.597(-2)$ & $9.438(-3)$ & $5.427(-3)$ & $3.056(-3)$ \\
$2^{-20}$ & $3.959(-2)$ & $2.589(-2)$ & $1.597(-2)$ & $9.438(-3)$ & $5.427(-3)$ & $3.056(-3)$ \\
\hline
\end{tabular}

Table 6: The maximum absolute errors of Example 6.6 for $\delta=\varepsilon / 10$.

\begin{tabular}{ccccccc}
\hline$\varepsilon \backslash \hbar$ & $h=2^{-5}$ & $h=2^{-6}$ & $h=2^{-7}$ & $h=2^{-8}$ & $h=2^{-9}$ & $h=2^{-10}$ \\
\hline $2^{-2}$ & $1.226(-2)$ & $8.036(-2)$ & $5.188(-3)$ & $3.637(-3)$ & $2.064(-3)$ & $1.073(-3)$ \\
$2^{-4}$ & $2.106(-2)$ & $1.502(-2)$ & $9.571(-3)$ & $6.337(-3)$ & $4.762(-3)$ & $4.040(-3)$ \\
$2^{-6}$ & $2.453(-2)$ & $1.986(-2)$ & $1.498(-2)$ & $9.678(-3)$ & $6.526(-3)$ & $5.236(-3)$ \\
$2^{-8}$ & $5.869(-2)$ & $1.846(-2)$ & $1.537(-2)$ & $1.347(-2)$ & $9.240(-3)$ & $6.160(-3)$ \\
$2^{-10}$ & $5.620(-2)$ & $2.939(-2)$ & $1.315(-2)$ & $1.044(-2)$ & $4.051(-3)$ & $6.183(-3)$ \\
$2^{-12}$ & $5.064(-2)$ & $2.459(-2)$ & $1.483(-2)$ & $8.703(-3)$ & $7.417(-3)$ & $6.402(-3)$ \\
$2^{-14}$ & $4.865(-2)$ & $2.146(-2)$ & $1.120(-2)$ & $7.504(-3)$ & $6.483(-3)$ & $5.871(-3)$ \\
$2^{-16}$ & $4.811(-2)$ & $2.048(-2)$ & $9.575(-3)$ & $7.131(-3)$ & $6.359(-3)$ & $5.816(-3)$ \\
$2^{-18}$ & $4.797(-2)$ & $2.022(-2)$ & $9.103(-3)$ & $7.110(-3)$ & $6.331(-3)$ & $5.804(-3)$ \\
$2^{-20}$ & $4.794(-2)$ & $2.016(-2)$ & $8.981(-3)$ & $7.104(-3)$ & $6.325(-3)$ & $5.801(-3)$ \\
& & & Results in $[10]$ & & \\
$2^{-2}$ & $3.196(-2)$ & $1.674(-2)$ & $8.569(-3)$ & $4.337(-3)$ & $2.182(-3)$ & $1.094(-3)$ \\
$2^{-4}$ & $8.124(-2)$ & $5.037(-2)$ & $2.977(-2)$ & $1.723(-2)$ & $9.793(-3)$ & $5.483(-3)$ \\
$2^{-6}$ & $9.471(-2)$ & $6.333(-2)$ & $3.741(-2)$ & $2.084(-2)$ & $1.139(-2)$ & $6.206(-3)$ \\
$2^{-8}$ & $8.867(-2)$ & $6.217(-2)$ & $3.909(-2)$ & $2.297(-2)$ & $1.278(-2)$ & $6.864(-3)$ \\
$2^{-10}$ & $8.486(-2)$ & $5.858(-2)$ & $3.671(-2)$ & $2.197(-2)$ & $1.284(-2)$ & $7.279(-3)$ \\
$2^{-12}$ & $8.366(-2)$ & $5.725(-2)$ & $3.531(-2)$ & $2.075(-2)$ & $1.198(-2)$ & $6.887(-3)$ \\
$2^{-14}$ & $8.334(-2)$ & $5.688(-2)$ & $3.487(-2)$ & $2.029(-2)$ & $1.153(-2)$ & $6.469(-3)$ \\
$2^{-16}$ & $8.325(-2)$ & $5.678(-2)$ & $3.476(-2)$ & $2.017(-2)$ & $1.139(-2)$ & $6.344(-3)$ \\
$2^{-18}$ & $8.323(-2)$ & $5.675(-2)$ & $3.473(-2)$ & $2.013(-2)$ & $1.135(-2)$ & $6.304(-3)$ \\
$2^{-20}$ & $8.323(-2)$ & $5.675(-2)$ & $3.472(-2)$ & $2.012(-2)$ & $1.134(-2)$ & $6.294(-3)$ \\
\hline
\end{tabular}


Table 7: The maximum absolute errors of Example 6.7 for $\delta=2 \varepsilon / 5$.

\begin{tabular}{ccccc}
\hline$\varepsilon \backslash \hbar$ & \multicolumn{1}{c}{$\mathrm{h}=2^{-7}$} & $\mathrm{~h}=2^{-8}$ & $\mathrm{~h}=2^{-9}$ & $\mathrm{~h}=2^{-10}$ \\
\hline $2^{-8}$ & $5.204(-4)$ & $2.304(-4)$ & $1.095(-4)$ & $5.359(-4)$ \\
$2^{-10}$ & $4.158(-4)$ & $1.706(-4)$ & $7.809(-5)$ & $3.757(-5)$ \\
$2^{-12}$ & $4.587(-4)$ & $1.288(-4)$ & $5.790(-5)$ & $2.652(-5)$ \\
$2^{-14}$ & $4.545(-4)$ & $1.488(-4)$ & $4.175(-5)$ & $1.936(-5)$ \\
$2^{-16}$ & $4.842(-4)$ & $1.500(-4)$ & $4.850(-5)$ & $1.390(-5)$ \\
$2^{-18}$ & $5.037(-4)$ & $1.574(-4)$ & $4.935(-5)$ & $1.586(-5)$ \\
$2^{-20}$ & $5.190(-4)$ & $1.637(-4)$ & $5.149(-5)$ & $1.624(-5)$ \\
& & \multicolumn{4}{c}{ Results in $[11]$} & \\
$2^{-8}$ & $9.804(-4)$ & $4.876(-4)$ & $2.363(-4)$ & $1.018(-4)$ \\
$2^{-10}$ & $9.864(-4)$ & $4.897(-4)$ & $2.444(-4)$ & $1.219(-4)$ \\
$2^{-12}$ & $9.933(-4)$ & $4.921(-4)$ & $2.446(-4)$ & $1.222(-4)$ \\
$2^{-14}$ & $1.794(-3)$ & $4.926(-4)$ & $2.451(-4)$ & $1.223(-4)$ \\
$2^{-16}$ & $1.388(-3)$ & $8.974(-4)$ & $2.460(-4)$ & $1.223(-4)$ \\
$2^{-18}$ & $9.948(-4)$ & $6.938(-4)$ & $4.488(-4)$ & $1.232(-4)$ \\
$2^{-20}$ & $9.948(-4)$ & $4.928(-4)$ & $3.468(-4)$ & $2.245(-4)$ \\
\hline
\end{tabular}

Table 8: The maximum absolute errors of Example 6.8 for $\delta=2 \varepsilon / 5$.

\begin{tabular}{cccccc}
\hline$\varepsilon \backslash \hbar$ & $h=2^{-6}$ & $h=2^{-7}$ & $h=2^{-8}$ & $h=2^{-9}$ & $h=2^{-10}$ \\
\hline \multicolumn{5}{c}{ Present method } \\
$2^{-8}$ & $1.2065(-2)$ & $2.342(-3)$ & $1.037(-3)$ & $4.929(-4)$ & $2.411(-4)$ \\
$2^{-10}$ & $1.0895(-2)$ & $1.871(-3)$ & $7.678(-3)$ & $3.514(-4)$ & $1.690(-4)$ \\
$2^{-12}$ & $1.0656(-2)$ & $2.064(-3)$ & $5.799(-3)$ & $2.605(-4)$ & $1.193(-4)$ \\
$2^{-14}$ & $1.0596(-2)$ & $2.045(-3)$ & $6.697(-3)$ & $1.878(-4)$ & $8.712(-5)$ \\
$2^{-16}$ & $1.0581(-2)$ & $2.179(-3)$ & $6.750(-3)$ & $2.182(-4)$ & $6.255(-5)$ \\
$2^{-18}$ & $1.0577(-2)$ & $2.267(-3)$ & $7.085(-3)$ & $2.220(-4)$ & $7.148(-5)$ \\
$2^{-20}$ & $1.0576(-2)$ & $2.290(-3)$ & $7.367(-3)$ & $2.317(-4)$ & $7.311(-5)$ \\
& \multicolumn{5}{c}{ Results in $[11]$} \\
$2^{-8}$ & $2.568(-2)$ & $1.274(-2)$ & $6.335(-3)$ & $3.064(-3)$ & $1.319(-3)$ \\
$2^{-10}$ & $2.576(-2)$ & $1.278(-2)$ & $6.366(-3)$ & $3.177(-3)$ & $1.585(-3)$ \\
$2^{-12}$ & $3.212(-2)$ & $1.279(-2)$ & $6.371(-3)$ & $3.179(-3)$ & $1.588(-3)$ \\
$2^{-14}$ & $2.987(-2)$ & $1.837(-2)$ & $7.031(-3)$ & $3.180(-3)$ & $1.588(-3)$ \\
$2^{-16}$ & $2.579(-2)$ & $1.695(-2)$ & $1.026(-2)$ & $3.900(-3)$ & $1.588(-3)$ \\
$2^{-18}$ & $2.579(-2)$ & $1.279(-2)$ & $9.401(-3)$ & $5.647(-3)$ & $2.133(-3)$ \\
$2^{-20}$ & $2.579(-2)$ & $1.279(-2)$ & $6.793(-3)$ & $5.136(-3)$ & $3.072(-3)$ \\
\hline
\end{tabular}

Table 9: CPU-running time for the Examples with $\mathrm{N}=100$.

\begin{tabular}{cc}
\hline & CPU running time \\
\hline Example 6.1 & 2.0625 (with $\varepsilon=0.01, \delta=0.8 \varepsilon$ ) \\
Example 6.2 & 1.8906 (with $\varepsilon=0.1, \delta=0.8 \varepsilon$ ) \\
Example 6.3 & 2.0156 (with $\varepsilon=0.01, \delta=0.025$ ) \\
Example 6.4 & 2.1563 (with $\varepsilon=0.1, \delta=0.8 \varepsilon$ ) \\
Example 6.5 & 1.9063 (with $\varepsilon=0.1, \delta=\frac{\varepsilon}{20}$ ) \\
Example 6.6 & 2.0313 (with $\varepsilon=0.1, \delta=\frac{\varepsilon}{20}$ ) \\
Example 6.7 & 2.0625 (with $\varepsilon=0.1, \delta=0.07 \varepsilon$ ) \\
Example 6.8 & 2.2188 (with $\varepsilon=0.1, \delta=0.07 \varepsilon$ ) \\
\hline
\end{tabular}

\section{References}

[1] R. Darzi, A. Neamaty, Y. Darzi, B. Mohammadzadeh, A Combined Method for the Numerical Solution of Boundary Value Problems of Second Order, J. Math. Comput. Sci., 4 (2012), 102-109. 1

[2] M. W. Derstine, H. M. Gibbs, F. A. Hopf, D. L. Kaplan, Bifurcation gap in a hybrid optically bistable system, Phys. Rev. A, 26 (1982), 3720-3722. 1

[3] E. P. Doolan, J. J. H. Miller, W. H. A. Schilders, Uniform Numerical Methods for Problems with Initial and Boundary Layers, Boole Press, Dublin, (1982). 1, 2.1, 2.2, 6 
[4] L. E. Él'sgol'st, S. B. Norkin, Introduction to the theory and application of differential equations with deviating arguments, Academic Press, New York-London, (1973). 1

[5] P. A. Farrell, A. F. Hegarty, J. J. H. Miller, E. O’Riordan, G. I. Shishkin, Robust Computational Techniques for Boundary Layers, Chapman \& Hall/CRC, Boca Raton, (2000). 1

[6] M. K. Kadalbajoo, K. K. Sharma, Numerical analysis of singularly perturbed delay differential equations with layer behavior, Appl. Math. Comput., 157 (2004), 11-28. 1, 1, 3

[7] C. G. Lange, R. M. Miura, Singular perturbation analysis of boundary value problems for differential-difference equations. II. Rapid oscillations and resonances, SIAM J. Appl. Math., 45 (1985), 687-707. 1

[8] C. G. Lange, R. M. Miura, Singular Perturbation Analysis of Boundary-Value Problems for Differential-Difference Equations V small shifts with layer behavior, SIAM J. Appl. Math., 54 (1994), 249-272. 1

[9] A. Longtin, J. Milton, Complex oscillations in the human pupil light reflex with mixed and delayed feedback, Math. Biosci., 90 (1988), 183-199. 1

[10] P. Rai, Numerical analysis of singularly perturbed differential-difference turning point problems Ph.D. Thesis, Punjab University, Chandigarh, India, (2013). 5, 6

[11] P. Rai, K. K. Sharma, Numerical method for singularly perturbed differential-difference equations with turning point, Int. J. Pure Appl. Math., 73 (2011), 451-470. 7, 8

[12] Y. N. Reddy, G. B. S. L. Soujanya, K. Phaneendra, Numerical Integration Method for Singularly Perturbed Delay Differential Equations, Int. J. Appl. Sci. Eng., 10 2012, 249-261. 2, 4

[13] G. B. S. L. Soujanya, Y. N. Reddy, Computational method for singularly perturbed delay differential equations with layer or oscillatory behaviour, Appl. Math. Infor. Sci., 10 (2016), 1-10. 1

[14] R. B. Stein, Some models of neuronal variability, Biophys. J., 7 (1967), 37-68. 1

[15] H. C. Tuckwell, Introduction to Theoretical Neurobiology, Cambridge University Press, Cambridge, (1988). 1 